\title{
ABSTRACTS SA HEART CONGRESS 2010
}

\section{Impact of pattern of left ventricular geometric adaptation on left ventricular ejection fraction in patients of African ancestry with moderate to severe hypertension}

\section{Haroon Abbasi, Elena Libhaber, Gavin Norton, Angela Woodiwiss, Karen Sliwa and Rafique Essop}

Division of Cardiology, Chris Hani Baragwanath Hospital, University of the Witwatersrand, Johannesburg, South Africa

Introduction: The pathophysiology of left ventricular (LV) remodelling in patients with hypertension (HT) is unclear.

Aim: To evaluate the impact of four different types of geometric adaptations to hypertension on left ventricular ejection fraction (LVEF).

Methods: A prospective single-centre study of 175 Black African patients (55\% female, age $=53.1 \pm 10.1$ years) with moderate-to-severe HT was performed. HT was defined as systolic or diastolic daytime ambulatory BP (ABPM) >140mmHg or 95mmHg respectively. Echocardiography was performed using a HP | 500 machine connected to $2.5 \mathrm{MHZ}$ transducer. LV dimensions (LVEDD, LVESD, LVEF) were measured using standard American Society of Echocardiography definitions. Comparisons between the four geometric patterns were analysed using the Kruskal-Wallis test.

Results:

Four patterns

\begin{tabular}{|c|c|c|c|c|c|}
\hline & $\begin{array}{l}\text { Normal } \\
\text { geometry }\end{array}$ & $\begin{array}{l}\text { Concentric } \\
\text { remodelling }\end{array}$ & $\begin{array}{c}\text { Concentric } \\
\text { LVH }\end{array}$ & Eccentric LVH & P value \\
\hline Age (years) & $54.02 \pm 9.30$ & $52.25 \pm 8.80$ & $51.30 \pm 11.62$ & $60.35 \pm 13.18$ & 0.075 \\
\hline Gender (f,n,\%) & $17(38)$ & $20(11)$ & $46(100)$ & $14(100)$ & $<0.000$ । \\
\hline SBP (din) & $163.5 \mid \pm 15.07$ & $171.37 \pm 21.80$ & $177.21 \pm 19.75$ & $172.42 \pm 17.61$ & 0.012 \\
\hline DBP (din) & $102.44 \pm 9.43$ & $105.00 \pm 10.49$ & $106.13 \pm 9.61$ & $105.35 \pm 10.59$ & 0.30 \\
\hline SBP (day) & $158.77 \pm 13.08$ & $166.08 \pm 15.39$ & $169.54 \pm 19.02$ & $165.35 \pm 15.84$ & 0.023 \\
\hline DBP (day) & $101.88 \pm 7.26$ & $106.64 \pm 8.96$ & $106.21 \pm 10.69$ & $|04.50 \pm 8.5|$ & 0.023 \\
\hline SBP (24-h) & $156.20 \pm 12.91$ & $161.57 \pm 16.00$ & $165.89 \pm 19.80$ & $|62.7| \pm 16.61$ & 0.059 \\
\hline DBP (24-h) & $98.86 \pm 7.50$ & $102.62 \pm 9.49$ & $103.02 \pm 11.25$ & $|00.92 \pm 8.5|$ & 0.08 \\
\hline SBP (din) & $163.5 \mid \pm 15.07$ & $171.37 \pm 21.80$ & $177.21 \pm 19.75$ & $|72.42 \pm 17.6|$ & 0.012 \\
\hline $\mathrm{DBP}$ (din) & $102.44 \pm 9.43$ & $105.00 \pm 10.49$ & $106.13 \pm 9.61$ & $105.35 \pm 10.59$ & 0.30 \\
\hline LVEDD & $5.03 \pm 0.60$ & $4.56 \pm 0.76$ & $4.41 \pm 0.49$ & $5.38 \pm 0.53$ & $<0.0001$ \\
\hline LVESD & $3.55 \pm 0.87$ & $3.12 \pm 0.78$ & $2.88 \pm 0.46$ & $3.80 \pm 0.56$ & $<0.0001$ \\
\hline EF \% & $57.12 \pm 16.69$ & $59.11 \pm 12.59$ & $63.60 \pm 8.03$ & $52.35 \pm 10.14$ & 0.0075 \\
\hline
\end{tabular}

Conclusion: Patients with eccentric LVH have the lowest systolic performance as measured by LVEF. These results suggest that patients with eccentric LVH need more aggressive therapy for heart failure. 


\section{Correlates of renal injury in patients of African ancestry with moderate to severe hypertension}

\section{H. Abbasi, E. Libhaber, G. Norton, A. Woodiwiss, T. Nunkoo, P. Gumede, N.R. Molefe, Z. Hlatshwayo, K. Sliwa and R. Essop}

Division of Cardiology, Chris Hani Baragwanath Hospital, University of the Witwatersrand, Johannesburg, South Africa

Background: In patients of African ancestry, hypertension is a frequent cause of kidney damage and end-stage renal disease.

Aim: We sought to identify office blood pressure (BP), ambulatory blood pressure (ABPM) and echocardiographic predictors of indices of renal injury.

Methods: Office blood pressure (dinamap), 24 hours ABPM using an Oscar 2 system and echocardiography using Sonos I 500 machine were performed in 175 patients (55\% female, age $53.1 \pm 10.1$ years). Moderate hypertension was defined as systolic or diastolic daytime ABPM > $140 \mathrm{mmHg}$ or $95 \mathrm{mmHg}$ respectively. 24 hours APBM was characterised as systolic and diastolic day, night and 24 hours reading and pulse pressure measured. Echo measurements were performed according to American Society of echocardiography definitions. LV mass was calculated. Renal indices included spot urine for microalbumin and micralbumin/creatinine ratio. Spearman correlation coefficient was adjusted for age and gender.

Results:

\begin{tabular}{|c|c|c|c|}
\hline & & $\mathbf{R}$ & P \\
\hline \multirow[t]{4}{*}{ Microalbumin (no I24) } & $\operatorname{SBP}(24 \mathrm{~h})$ & 0.24 & 0.0067 \\
\hline & PP (day) & 0.24 & 0.008 \\
\hline & PP $(24 h)$ & 0.20 & 0.02 \\
\hline & LVMI $\left(\mathrm{g} / \mathrm{m}^{2}\right)$ & 0.18 & 0.04 \\
\hline \multirow[t]{4}{*}{ Microalb/creat ratio (no 124) } & $\operatorname{SBP}(24 h)$ & 0.23 & 0.01 \\
\hline & PP (day) & 0.25 & 0.006 \\
\hline & PP $(24 h)$ & 0.21 & 0.018 \\
\hline & LVMI $\left(\mathrm{g} / \mathrm{m}^{2}\right)$ & 0.22 & 0.014 \\
\hline
\end{tabular}

Conclusion: Daytime pulse pressure appears to be most closely correlated with renal injury. Systolic blood pressure correlates better than diastolic blood pressure with renal damage. There is close correlation between LVMI and urine micralbumin creatinine ratio. 


\section{Prevalence of left ventricular geometric phenotype in patients of African ancestry with moderate to severe hypertension}

\section{Haroon Abbasi, Elena Libhaber, Gavin Norton, Angela Woodiwiss, Karen Sliwa and Rafique Essop}

Division of Cardiology, Chris Hani Baragwanath Hospital, University of the Witwatersrand, Johannesburg, South Africa

Background: Hypertension (HT) is commonly associated with left ventricular (LV) structural remodelling, but prevalence of different types of geometric changes in South African population of African ancestry with moderate to severe hypertension is unknown.

Objective: To determine four different geometric patterns using echocardiography in patients of African ancestry with moderate to severe hypertension.

Design and methods: A single-centre study of 175 Black African patients (55\% female, age $=53.1 \pm 10.1$ years) with moderate-to-severe HT was performed. All measurements were made using standard American Society of Echocardiography definitions. LVH as LV mass index $>120 \mathrm{~g} / \mathrm{m}^{2}$ in males and $>100 \mathrm{~g} / \mathrm{m}^{2}$ in females. For relative wall thickness (RWT) cut point of 0.44 was used. Four groups were defined: ( I) Normal geometry; (2) Concentric remodelling; (3) Concentric left ventricular hypertrophy (LVH); and (4) Eccentric LVH. HT was defined as systolic or diastolic daytime ambulatory BP (ABPM) $>140 \mathrm{mmHg}$ or $95 \mathrm{mmHg}$ respectively. Values are expressed as mean \pm SD for all variables. Comparisons between the four geometric patterns were analysed using Kruskal-Wallis test.

Results:

Baseline and echocardiographic characteristics of the patients

\begin{tabular}{|c|c|c|c|c|c|}
\hline & $\begin{array}{l}\text { Normal } \\
\text { geometry }\end{array}$ & $\begin{array}{l}\text { Concentric } \\
\text { remodelling }\end{array}$ & $\begin{array}{c}\text { Concentric } \\
\text { LVH }\end{array}$ & Eccentric LVH & P value \\
\hline Number (\%) & $45(26)$ & $70(40)$ & $46(26)$ & $14(8)$ & \\
\hline Age (years) & $54.02 \pm 9.30$ & $52.25 \pm 8.80$ & $51.30 \pm 11.62$ & $60.35 \pm 13.18$ & 0.075 \\
\hline Gender (f,n,\%) & $17(38)$ & $20(11)$ & $46(100)$ & $14(100)$ & $<0.000$ । \\
\hline $\mathrm{BMI}\left(\mathrm{kg} / \mathrm{m}^{2}\right)$ & $30.85 \pm 7.1$ & $30.34 \pm 4.81$ & $31.53 \pm 5.66$ & $32.52 \pm 8.05$ & 0.61 \\
\hline Diabetic (n,\%) & $6(13)$ & $6(9)$ & $11(24)$ & $3(21)$ & \\
\hline SBP (din) & $163.51 \pm 15.07$ & $171.37 \pm 21.80$ & $177.21 \pm 19.75$ & $172.42 \pm 17.6 \mid$ & 0.012 \\
\hline DBP (din) & $102.44 \pm 9.43$ & $105.00 \pm 10.49$ & $106.13 \pm 9.61$ & $105.35 \pm 10.59$ & 0.30 \\
\hline SBP (day) & $158.77 \pm 13.08$ & $166.08 \pm 15.39$ & $169.54 \pm 19.02$ & $165.35 \pm 15.84$ & 0.023 \\
\hline DBP (day) & $101.88 \pm 7.26$ & $106.64 \pm 8.96$ & $106.21 \pm 10.69$ & $|04.50 \pm 8.5|$ & 0.023 \\
\hline SBP (night) & $149.04 \pm 14.06$ & $153.77 \pm 19.11$ & $160.30 \pm 23.30$ & $156.42 \pm 16.78$ & 0.07 \\
\hline DBP (night) & $91.93 \pm 10.04$ & $95.07 \pm 11.70$ & $96.43 \pm 12.79$ & $93.71 \pm 11.22$ & 0.08 \\
\hline SBP (24-h) & $156.20 \pm 12.90$ & $161.57 \pm 16.00$ & $165.89 \pm 19.80$ & $|62.7| \pm|6.6|$ & 0.059 \\
\hline DBP (24-h) & $98.86 \pm 7.50$ & $102.62 \pm 9.49$ & $103.02 \pm 11.25$ & $100.92 \pm 8.51$ & 0.08 \\
\hline PWED & $0.93 \pm 0.16$ & $1.31 \pm 0.26$ & $1.34 \pm 0.25$ & $0.96 \pm 0.09$ & $<0.0001$ \\
\hline $\mathrm{EF} \%$ & $57.12 \pm 16.69$ & $59.11 \pm 12.59$ & $63.60 \pm 8.03$ & $52.35 \pm 10.14$ & 0.00075 \\
\hline LVMI $\left(g / m^{2}\right)$ & $105.80 \pm 34.65$ & $|30.6| \pm 47.65$ & $137.87 \pm 28.63$ & $134.85 \pm 21.57$ & $<0.0001$ \\
\hline RWT & $0.37 \pm 0.06$ & $0.59 \pm 0.16$ & $0.61 \pm 0.14$ & $0.36 \pm 0.04$ & $<0.000$ । \\
\hline
\end{tabular}

Conclusion: As opposed to other population groups, the predominant geometric phenotype in patients of African ancestry with moderate to severe hypertension is concentric remodelling and concentric LVH. Apparently there is no significant blood pressure (BP) gradient across three different groups except in patients with normal geometry who has lower BP. The explanation of a unique pattern of LV geometry in patients of African ancestry needs further investigation. 


\section{Relationship between left ventricular geometry pattern and diastolic function in patients}

of African ancestry with moderate to severe hypertension

\section{Haroon Abbasi, Elena Libhaber, Gavin Norton, Angela Woodiwiss, Karen Sliwa and Rafique Essop}

Division of Cardiology, Chris Hani Baragwanath Hospital, University of the Witwatersrand, Johannesburg, South Africa

Background: Based on left ventricular mass index (LVMI) and relative wall thickness (RWT), four patterns of geometric adaptation may be observed in patients with hypertension (HT).

Aim: To determine the relationship between the patterns of LV geometric response and diastolic abnormalities in patients with moderateto-severe hypertension.

Design and methods: A single-centre study of 175 Black African patients (55\% female, age $53.1 \pm 10.1$ years) with moderate-to-severe HT. HT was defined as systolic or diastolic daytime ambulatory BP (ABPM) $>140 \mathrm{mmHg}$ or $95 \mathrm{mmHg}$ respectively. Using echocardiography, patients were grouped as follows: (I) Normal LV geometry; (2) Concentric LV remodelling; (3) Concentric LV hypertrophy; and (LVH) (4) Eccentric $\mathrm{LVH}$. All measurements were made using standard American Society of Echocardiography definitions. LVH was defined as LVMI > I20g/m² in males and $>100 \mathrm{~g} / \mathrm{m}^{2}$ in females. RWT was calculated by formula $(2 \times$ PWT/ LVEDD). LV mass and LV relative wall thickness were calculated to classify patients into four different geometric groups. Pulsed wave Doppler was used to measure transmitral inflow velocities (E,A, E/A ratio) and abnormalities in diastolic function. Values are expressed as mean + SD for all variables. Comparisons between the four geometric patterns were analysed using the Kruskal-Wallis test.

Results:

Blood pressure and echo measurements of patients

\begin{tabular}{|c|c|c|c|c|c|}
\hline & $\begin{array}{l}\text { Normal } \\
\text { geometry }\end{array}$ & $\begin{array}{l}\text { Concentric } \\
\text { remodelling }\end{array}$ & $\begin{array}{c}\text { Concentric } \\
\text { LVH }\end{array}$ & Eccentric LVH & P value \\
\hline SBP (din) & $163.5 \mid \pm 15.07$ & $171.37 \pm 21.80$ & $177.21 \pm 19.75$ & $172.42 \pm 17.6 \mid$ & 0.012 \\
\hline DBP (din) & $102.44 \pm 9.43$ & $105.00 \pm 10.49$ & $106.13 \pm 9.61$ & $105.35 \pm 10.59$ & 0.30 \\
\hline SBP (day) & $158.77 \pm 13.08$ & $166.08 \pm 15.39$ & $169.54 \pm 19.02$ & $165.35 \pm 15.84$ & 0.023 \\
\hline DBP (day) & $101.88 \pm 7.26$ & $106.64 \pm 8.96$ & $106.21 \pm 10.69$ & $104.50 \pm 8.51$ & 0.023 \\
\hline SBP (24-h) & $156.20 \pm 12.91$ & $161.57 \pm 16.00$ & $165.89 \pm 19.80$ & $|62.7| \pm 16.61$ & 0.059 \\
\hline DBP (24-h) & $98.86 \pm 7.50$ & $102.62 \pm 9.49$ & $103.02 \pm 11.25$ & $100.92 \pm 8.51$ & 0.08 \\
\hline LVEDD & $5.03 \pm 0.60$ & $4.56 \pm 0.76$ & $4.41 \pm 0.49$ & $5.38 \pm 0.53$ & $<0.000$ । \\
\hline LVESD & $3.55 \pm 0.87$ & $3.12 \pm 0.78$ & $2.88 \pm 0.46$ & $3.80 \pm 0.56$ & $<0.000$ I \\
\hline SEPED & $1.14 \pm 0.31$ & $1.36 \pm 0.37$ & $1.44 \pm 0.35$ & $1.25 \pm 0.18$ & 0.0002 \\
\hline PWED & $0.93 \pm 0.16$ & $1.31 \pm 0.26$ & $1.34 \pm 0.25$ & $0.96 \pm 0.09$ & $<0.000$ । \\
\hline $\mathrm{EF} \%$ & $57.12 \pm 16.69$ & $59.11 \pm 12.59$ & $63.60 \pm 8.03$ & $52.35 \pm 10.14$ & 0.0075 \\
\hline LVM (g) & $202.03 \pm 67.89$ & $252.57 \pm 102.40$ & $245.88 \pm 59.29$ & $242.34 \pm 41.63$ & 0.0029 \\
\hline LVMI $\left(g / \mathrm{m}^{2}\right)$ & $105.80 \pm 34.65$ & $|30.6| \pm 47.65$ & $137.87 \pm 28.63$ & $134.85 \pm 21.57$ & $<0.000$ I \\
\hline RWT & $0.37 \pm 0.06$ & $0.59 \pm 0.16$ & $0.61 \pm 0.14$ & $0.36 \pm 0.04$ & $<0.000$ I \\
\hline MFS & $0.19 \pm 0.07$ & $0.18 \pm 0.05$ & $0.20 \pm 0.06$ & $0.20 \pm 0.06$ & 0.60 \\
\hline E-wave (ms) & $69.75 \pm 20.71$ & $71.15 \pm 21.02$ & $61.63 \pm 16.21$ & $63.34 \pm 23.93$ & 0.16 \\
\hline A-wave (ms) & $71.06 \pm 17.61$ & $70.25 \pm 18.67$ & $74.92 \pm 25.77$ & $74.93 \pm 18.43$ & 0.62 \\
\hline E/A ratio $(n=42 / 64 / 37 / 13)$ & $1.06 \pm 0.48$ & $1.10 \pm 0.50$ & $0.91 \pm 0.39$ & $0.94 \pm 0.62$ & 0.15 \\
\hline
\end{tabular}

Conclusion: Using transmitral inflow velocities there does not appear to be any significant difference in diastolic function in the four groups of geometric adaptation. More sensitive techniques may be required to detect diastolic function. 


\section{Sirtuin I: A cardioprotective target for the aged heart?}

\section{Tasneem Adam, Stephanie Sharp, Lionel H. Opie and Sandrine Lecour}

Hatter Cardiovascular Research Institute, Faculty of Health Sciences, University of Cape Town, South Africa

Introduction: Ischaemic preconditioning (IPC) is a powerful protective approach for reducing myocardial damage. Interestingly, the cardioprotective effect of IPC is lost with age. Signalling molecules involved in preserving IPC's effectiveness, with ageing, is unclear. Sirtuin I (Sirt I) is a class III histone deacetylase with diverse biological functions, including longevity and oxidative stress resistance. We propose that Sirt I contributes to IPC-mediated cardioprotection, and that IPC's beneficial effect is lost with age due to a decrease in Sirt I activity.

Methods: Isolated young ( 9 weeks) and aged (12 months) rat hearts were subjected to 30 min global ischaemia and Ihr reperfusion. Preconditioning was induced by $2 \times 5$ min cycles of ischaemia reperfusion (IPC), or with resveratrol ( $10 \mu M$, Sirtuin activator) for 15 min before

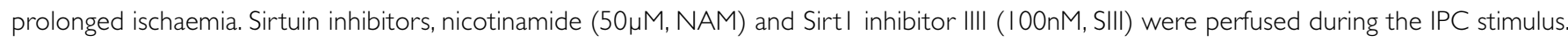
Haemodynamic parameters and Sirt I activity in nuclear extracts were measured as endpoints ( $\mathrm{n}=6$ for all groups).

Results: IPC (43.5 $\pm 3.1 \%)$ and resveratrol $(32.8 \pm 4.6 \%)$ improved functional recovery compared to young control hearts (I6.6 $\pm 2.4 \%$; $\mathrm{p}<0.05$ ). In contrast, aged hearts could not be preconditioned by either ischaemia or resveratrol ( $19.9 \pm 4.3 \%$ for IPC, $6.58 \pm 2.4 \%$ for resveratrol versus $13.9 \pm 2.9 \%$ for control). NAM administration abrogated the functional recovery of young hearts by $26.9 \%$, whereas $\mathrm{SIII}$ did not abolish the cardioprotective effect of IPC. Aged hearts ( $0.63 \pm 0.2$ arbitrary units) displayed significantly higher Sirt I activity than young hearts $(0.18 \pm 0.1$ arbitrary units; $p<0.05)$. However, IPC did not alter Sirt I activity in young hearts.

Conclusion: These data strongly suggest that loss of IPC-induced cardioprotection with ageing is independent of Sirt I activation.

\section{Transcatheter closure of a large coronary cameral fistula in a young boy}

\section{Paul Adams, Gcina Dumani and Antoinette Cilliers}

Division of Paediatric Cardiology, Department of Paediatrics and Child Health, Chris Hani Baragwanath Hospital, University of the Witwatersrand, Johannesburg, South Africa

Introduction: A coronary arterial fistula is an abnormal communication arising from a coronary artery which ends in a cardiac chamber or great vessel. These anomalies are rare and the majority are congenital. They often remain asymptomatic. Despite this they have a number of serious complications including sudden death, which can be avoided by occlusion of the fistulae.

Case description: We present a thirteen year old boy who presented with deteriorating effort tolerance and dyspnoea seven years after the incidental diagnosis of an innocent murmur. Examination was completely normal except for the presence of a 3/6 continuous murmur at the right parasternal border in the 4th and 5th intercostal spaces. Echocardiography revealed a large, abnormal communication between the left coronary artery and the right atrium consistent with the diagnosis of a coronary cameral fistula. Cardiac catheterisation and device closure was undertaken. Due to the tortuous nature of the fistula and the small right atrial opening an arterio-venous loop was required to allow retrograde placement of a device in the distal end of the fistula. A $20 \mathrm{~mm}$ Amplatzer vascular plug II was used to occlude the communication. No complications were experienced.

Conclusion: Coronary cameral fistulae are rare but potentially devastating anomalies if large and left untreated. Percutaneous device occlusion of the fistula proved to be safe and effective in our patient. 


\section{What causes Left Atrial Spontaneous Echocardiography Contrast (LASEC)? Is it stasis, turbulence or both?}

\section{S. Ahmed, H.P. Cyster, P.G. Herbst and A.F. Doubell}

Division of Cardiology, Department of Medicine, University of Stellenbosch and Tygerberg Hospital, South Africa

The formation of LASEC in patients with severe mitral stenosis (MS) is considered to be due to blood stasis in a dilated left atrium. Although stasis is undoubtedly required for the LASEC phenomenon, observations in our unit indicate that the visibility of LASEC seems to be enhanced by turbulent blood flow. Spontaneous echocardiography contrast (SEC) increases as blood accelerates towards the stenotic mitral valve and becomes manifest in the left ventricle as a discrete jet when blood accelerates through the narrowed valve.

We hypothesize that stasis is a fundamental pre-requisite for LASEC as it changes the properties of the blood components (e.g. clotting factors, platelet adherence and rouleaux formation) but requires turbulent flow to become manifest. This would imply that LASEC is not merely a phenomenon explained by the interaction of ultrasound waves with slow flowing blood. Rather it is caused by ultrasound waves interacting with blood which has been changed by stasis.

We report an index case, which demonstrates the enhanced visualisation of LASEC in areas of turbulent blood flow Based on the index case we have initiated a study to verify and quantify our hypothesis in 10 patients by comparing velocities in the areas displaying LASEC with areas where LASEC is not displayed.

Our observation implies that thromboembolic risk in patients with LASEC may be related to both stasis and its change of blood components. The exact change effected by stasis requires further study as this may reveal new treatment targets for prevention of thromboembolism.

\section{Markers of inflammation and endothelial activation in Black South Africans with HIV and Acute Coronary Syndromes}

\section{Anthony Becker, Elena Libhaber, Karen Sliwa, Simon Stewart and Rafique Essop \\ Department of Cardiology, Chris Hani Baragwanath Hospital, University of the Witwatersrand, Johannesburg, South Africa}

Background: HIV infection is associated with a pro-inflammatory and thrombophilic state but little is known about the link between inflammation and thrombosis in treatment-naïve patients with acute coronary syndromes (ACS).

Methods and results: Prospective single centre study from Soweto, South Africa, comparing markers of inflammation and endothelial cell activation in highly active anti-retroviral therapy-naïve HIV positive and negative patients presenting with ACS. Between March 2004 and February 2008, 30 consecutive Black South African HIV patients with ACS were compared to 30 Black HIV negative patients with ACS. The HIV patients were younger (43 \pm 7 vs. $54 \pm 13, p=0.004)$ and besides smoking (73\% vs. 33\%, $p=0.002)$ and lower HDL levels ( $0.8 \pm 0.3$ vs. $1.1 \pm 0.4, p=0.001)$ had fewer risk factors than the control group. At baseline, HIV patients had higher levels of tumour necrosis factor-a [5.8 (I.7- I5.0) vs. 0.19 (0.19-19.8) ng/mL, p=0.0004] and vascular cell adhesion molecule- I [263.3 (0.38-778.5) vs. I5I.3 (80.6-4I6.3) ng/mL, $\mathrm{p}=0.007$ ] compared to HIV negative patients as well as higher levels of macrophage chemoattractant protein-I at six months [70 (30-I 30) vs. $50(30-90) \mathrm{ng} / \mathrm{L}, \mathrm{p}=0.004]$.

Conclusion: Treatment-naïve Black South African patients with HIV and ACS have evidence of a pro-inflammatory state and greater degree of endothelial cell activation compared to HIV negative patients, both of which may play a direct role in the pathogenesis of ACS in this otherwise low risk population. MCP-I may play an important role in HIV-associated coronary artery disease. 


\title{
Antiphospholipid antibodies in Black South Africans with HIV and Acute Coronary Syndromes: Prevalence and clinical correlates
}

\author{
Anthony Becker, Elena Libhaber, Karen Sliwa, Sham Singh, Simon Stewart, Mohamed Tikly and Rafique Essop \\ Department of Cardiology, Chris Hani Baragwanath Hospital, University of the Witwatersrand, Johannesburg, South Africa
}

Background: HIV infection is associated with a high prevalence of antiphospholipid antibodies (aPL) and increased thrombotic events but the aetiopathogenic link between the two is unclear.

Methods and results: Prospective single centre study from Soweto, South Africa, comparing the prevalence of aPL in highly active anti-retroviral therapy (HAART) naïve HIV positive and negative patients presenting with Acute Coronary Syndromes (ACS). Between March 2004 and February 2008, 30 consecutive Black South African HIV patients with ACS were compared to 30 Black HIV negative patients with ACS. The HIV patients were younger (43 \pm 7 vs. $54 \pm 13, p=0.004)$ and besides smoking $(73 \%$ vs. 33\%, $p=0.002)$ and lower HDL levels (0.8 \pm 0.3 vs. $\mathrm{I} . \mathrm{I} \pm 0.4, \mathrm{p}=0.00 \mathrm{I}$ ) had fewer risk factors than the control group. HIV patients had a higher prevalence of anticardiolipin (aCL) IgG (47\% vs. $10 \%, p=0.003$ ) and anti-prothrombin (aPT) IgG antibodies ( $87 \%$ vs. $21 \%, p<0.001$ ), but there was no difference in the prevalence of the antiphospholipid syndrome (44\% vs. $24 \%, p=N / S$ ) and aPL were not predictive of clinical or angiographic outcomes.

Conclusion: Treatment-naïve Black South African HIV patients with ACS are younger with fewer traditional coronary risk factors than HIV negative patients but have a higher prevalence and different expression of aPL which is likely to be an epiphenomenon of the HIV infection rather than causally linked to thrombosis and the pathogenesis of ACS.

\section{Sarcomeric modifiers of hypertrophy in hypertrophic cardiomyopathy}

\author{
Liezl Bloem, Lize van der Merwe, Miriam Revera, Craig Kinnear, Marshall Herandien, Althea Goosen, Paul Brink \\ and Johanna Moolman-Smook \\ University of Stellenbosch Medical Research Centre (US/MRC), Centre for Molecular and Cellular Biology, Health Sciences, \\ University of Stellenbosch, South Africa
}

\begin{abstract}
Left ventricular hypertrophy $(\mathrm{LVH})$ is an independent predictor of risk of death and is a feature of common, multi-factorial disorders including diabetes and hypertension. Understanding the mechanisms leading to LVH is thus important, however investigating them in such complex disorders is challenging. Therefore, we have undertaken to unravel the molecular mechanisms of LVH in hypertrophic cardiomyopathy (HCM), an autosomal dominantly inherited disorder in which LVH is the primary feature. Interestingly, in HCM, LVH is extremely variable even in patients carrying the same HCM-causing mutation suggesting that additional modifying factors, possibly genetic, may influence the phenotype. Myosin heavy chain 7 (MYH7) encodes cardiac myosin, a myofilament involved in both sarcomere structure and contraction. Its crucial function together with its documented role as an HCM-causing gene, suggests that it is a plausible candidate modifier gene.

The investigation aimed to determine whether MYH7 is an HCM modifying gene by means of ABI validated Taqman SNP genotyping and family-based association testing with QTDT for comparison of genotypes to various hypertrophy traits. A total of 227 individuals, belonging to 22 HCM families, carrying one of three known founder HCM-causing mutations (R92WTNNT2, R403WMYH7, and A797TMYH7), was genotyped.

Statistically significant association was found for two of the SNPs investigated ( $p=0.004$ : rs2754 I 63; left ventricular mass (LVMecho); $p=0.016$ : rs2277475; principle component score (compl)). The study indicates the dual contribution of MYH7 to the pathogenesis of HCM as both causative and modifying and so furthers our understanding of cardiac hypertrophy development and highlights avenues for future therapeutic intervention studies.
\end{abstract}




\section{Evaluation of near-infrared spectroscopy in patients with acute coronary syndrome undergoing on- and off-pump coronary artery bypass graft surgery}

\section{Lezelle Botes, Lindie Liebenberg, Francis Edwin Smit and William Morris Leonard Neethling}

School of Health Technology, Central University of Technology, Bloemfontein, South Africa

Introduction: Central Nervous System (CNS) dysfunction continues to be a major cause of morbidity in Coronary Artery Bypass Graft (CABG) patients. The Somanetics ${ }^{\circledR}$ INVOS ${ }^{\circledR}$ Cerebral Oximeter provides continuous regional oxygen saturation ( $\left.\mathrm{rSO} 2\right)$ values. The aim of the study was to evaluate the relationship between cerebral rSO2 values (patients presenting with acute coronary syndrome (ACS)) during onand off-pump CABG surgery and post-operative neurological and clinical outcomes.

Methodology: An observational cross-sectional analytical study was conducted. Sixty patients diagnosed with ACS undergoing CABG surgery were recruited (30 off-pump and 30 on-pump). Analysis I: Cerebral regional oximetry values were compared between the on- and off-pump groups. Analysis 2: Patients were divided into two groups depending on their NIRS values. Group I had NIRS values indicating adequate circulation and group 2 values of impaired regional circulation (NIRS <50, $>20 \%$ drop from baseline). Intra-operative haemodynamics, renal function and post-operative complications were compared between the two groups.

Results: Analysis I: No difference in cerebral regional O2 could be demonstrated between on- and off-pump CABG techniques ( $p>0.05)$. Analysis 2: $13.3 \%(n=4)$ on-pump CABG patients versus $83.3 \%(n=25)$ off-pump surgery patients had adequate perfusion. Impaired circulation occurred in $86.7 \%(n=26)$ of on-pump patients and in $16.7 \%(n=5)$ of off-pump patients. No difference could be demonstrated in clinical and neurological outcomes between the groups.

Conclusion: No difference in regional cerebral perfusion could be demonstrated between on- and off-pump CABG surgery patients. No adverse clinical and neurological outcomes could be linked to short periods of impaired cerebral flow.

\section{The influence of pulmonary regurgitation on regional right ventricular function in children after surgical repair of tetralogy of Fallot}

\section{Stephen Brown, Benedicte Eyskens and Marc Gewillig}

Department of Pediatric Cardiology, University of the Free State, Bloemfontein, South Africa

Background: Pulmonary regurgitation after repair of tetralogy of Fallot (TOF) results in right ventricular (RV) dysfunction with significant late morbidity and mortality. The aim was to assess the influence of pulmonary regurgitation on regional RV function.

Patients and methods: In 48 asymptomatic children (age II.I \pm 3.3 years) with repair of TOF, peak systolic strain rate (SR) and endsystolic strain (e) were acquired using Colour Doppler Myocardial Imaging. Right ventricular indices were quantified by magnetic resonance imaging. Echocardiography and exercise capacity was also performed.

Results: Forty complete datasets were analysed. An inverse linear relationship was demonstrated between the degree of pulmonary regurgitation and right ventricle end-systolic e $(r=-0.53, p<0.0 \mathrm{I})$ as well as a correlation with peak systolic SR $(r=-0.35, \mathrm{p}<0.0 \mathrm{I})$. A correlation existed between peakVO2 and peak systolic SR ( $r=0.5 \mathrm{I}, \mathrm{p}=0.00 \mathrm{I})$ and end-systolic e $(r=0.33, \mathrm{p}<0.05)$.

Conclusion: In asymptomatic children after repair of TOF, pulmonary regurgitation is associated with impaired regional systolic RV deformation indices. Regional strain and strain rate may be an early indicator of RV dysfunction in patients with post TOF and pulmonary regurgitation. 


\section{Sutureless rescue of a severely hypoplastic pulmonary artery}

\section{Stephen Brown and Marc Gewillig}

Department of Pedaitric Cardiology, University of the Free State, Bloemfontein, South Africa

The management of severely hypoplastic pulmonary arteries in the adolescent or adult with congenital heart disease is challenging. A patient with pulmonary atresia and ventricular septal defect (PA-VSD) presented with severe cyanosis due to a kinked and thrombosed right ventricle to pulmonary artery conduit. Retrograde wedge angiography showed a patent right and a diminutive left pulmonary artery deep in the lung hilus. Treatment options were limited and therefore a different strategy had to be considered in order to rescue the lung segments. This case report describes the novel use of a covered stent to successfully obtain a sutureless connection between a vascular graft and a diminutive pulmonary artery during a hybrid procedure.

\section{Use of a microcatheter in a telescopic system to reach difficult targets in complex congenital heart disease}

\section{Stephen Brown and Marc Gewillig}

Department of Pedaitric Cardiology, University of the Free State, Bloemfontein, South Africa

Background: Some lesions can be very difficult to reach, especially if acute angles and/or multiple turns "protect" access. Once reached, the guiding system needs to give sufficient support for balloons or stents to be deployed.

Methods: A "telescopic" system was created consisting of:

- a microcatheter with guidewire. This system fits into any catheter allowing a 0.035 " wire;

a $4 \mathrm{~F}$ "delivery" catheter; and

- if, sharp angles were encountered, the 4Fr catheter was deployed through a $6 \mathrm{Fr}$ or larger angulated guiding sheath. This was cut-off I $5 \mathrm{~cm}$ out of the groin and re-valvulated with a standard short introducer sheath.

Patients \& results: Since 2004, 89 microcatheter telescopic systems were used during a 4-year period in I 225 procedures (7.3\%). The technique allowed probing with a floppy steerable and exchangeable guidewire, securing any gained position as well as exchanges with a stiffer guide wire if required. Procedures where the telescopic system was effective included: Complex stenoses e.g. aortopulmonary collaterals $(n=21)$; tortuous ducts $(n=9)$; anterograde balloon dilation of critical aortic stenosis $(n=8)$; and crossing Blalock Taussig shunts ( $n=3)$, Once in place, the telescopic system allowed delivery of embolic material $(n=38)$, balloon angioplasty $(n=21)$, stent deployment $(n=23)$, fulguration of pulmonary valve $(n=1)$ or introduction of medication (1). The interventionalists felt that using the telescopic system had reduced fluoroscopy and procedure time.

Conclusion: The microcatheter-telescopic system is an invaluable tool to reach difficult targets and allows exchange for suitable guidewires permitting balloons, stents, embolisation material or radiofrequency energy to be deployed in such targets. This approach has become our standard when dealing with difficult targets protected by a tortuous route. 


\title{
Angiotensin II type 2 receptor gene polymorphism modulates extent of hypertrophy in hypertrophic cardiomyopathy
}

\author{
Nadia Carstens, Lize van der Merwe, Miriam Revera, Marshall Heradien Althea Goosen, Paul A. Brink and \\ Johanna Moolman-Smook \\ University of Stellenbosch Medical Research Council (US/MRC), Centre for Molecular and Cellular Biology, \\ University of Stellenbosch, South Africa
}

Hypertrophic cardiomyopathy (HCM), an inherited primary cardiac disorder mostly caused by defective sarcomeric proteins, serves as a model to investigate left ventricular hypertrophy $(\mathrm{LVH})$. The disease manifests extreme variability in the degree and pattern of LVH, even in HCM patients with the same causal mutation. Previous studies identified genes coding for renin-angiotensin-aldosterone system (RAAS) components as hypertrophy modifiers in HCM. Many of these investigations focussed on the angiotensin (Ang) II type I receptor (ATIR). However, Ang II is also capable of binding to Ang II type 2 receptors (AT2R) with anti-hypertrophic effects.

We investigated the effect of the functional + 1675 G/A polymorphism (rs | 403543) and additional single nucleotide polymorphisms (SNPs) in the 3' untranslated region of the AT2R gene (AGTR2) on a heritable composite hypertrophy score in an HCM family-cohort in which HCM founder-mutations segregate.

We report a significant association between the previously identified functional + I675 G/A polymorphism (rs I403543) and the hypertrophy score, independent of the primary HCM causal mutation, blood pressure and other hypertrophy covariates $(P=0.02)$. We estimate that each $A$ allele decreases the average cardiac wall thickness by $0.5 \mathrm{~mm}$ after taking known confounders into account. Given the functionality of $+1675 \mathrm{G} / \mathrm{A}$, it is possible to speculate that AT2R plays a cardioprotective role by modulating the development of hypertrophy in HCM, independent of blood pressure and the disease-causing mutation. This study further implies that +1675 G/A could potentially be used in a panel of markers that profile a genetic predisposition to LVH in HCM patients.

\section{The role of modified Maze radiofrequency procedure in patients with mega-atria and concomitant valvular heart disease}

\section{R.F. Chauke and J.L. Dongo}

Department of Cardiothoracic Surgery, University of Limpopo, Medunsa, South Africa

Define: (a) conversion to sinus rhythm after operation and (b) atrial fibrillation (AF) free survival at one year duration. Introduction: Valvular heart disease is frequently as a result of rheumatic heart disease in our setting and the patients are usually young and present late. We, therefore report on 25 patients who underwent modified Maze radiofrequency procedure using Medtronic radiofrequency ablation device, with concomitant valvular operations. All patients presented with permanent AF which is defined as AF lasting more than 4 weeks, does not self terminate and does not respond to cardioversion. Three patients were in NYHA class I, 7 patients in class II, I 3 in class III and 2 in class IV. Mega-atrium was defined as any atrium larger than $60 \mathrm{~mm}$.

Methods: Patients were divided into two groups. Group I were patients with LA diameter less than 60mm and Group 2 were patients with LA diameter of $60 \mathrm{~mm}$ or more. The procedures were done between November 2006 to August 2009. They were followed up for a period of a year. The patients were evaluated for symptomatic improvement, rhythm with ECG documentation and 2D echocardiography. Five patients were in Group I and 20 patients in Group 2. RF maze procedure was deemed successful when there was persistence of sinus rhythm without the use of anti-arrythmic drugs or electrical cardioversion after 7 days post-op.

Results: Eighteen patients were in sinus rhythm immediately post operatively. Sixteen in Group 2 and 4 in Group I. Two patients in Group 2 remained in atrial fibrillation, 3 died immediately post-operatively and the records of two other patients disappeared. The success rate being $78 \%$ after one year with $13 \%$ mortality not related to ablation.

Conclusion: It does appear that modified Maze RF may be beneficial to patients with mega-atria and concomitant valvular heart disease. The numbers are small to make a meaningful conclusion but the study is ongoing. 


\section{Audit of referrals for fetal echocardiography: 10 years in a tertairy centre}

\section{Sally-Ann Clur, Peter Mathijs van Brussel, M. Mathijssen, Jaap Ottenkamp and Caterina Madelena Bilardo}

Department of Pediatric Cardiology, Emma Children's Hospital, Academic Medical Centre (AMC) and Centre for Congenital Heart Anomalies Amsterdam-Leiden (CAHAL), The Netherlands

Objectives: As ultrasonographic skills improve, referrals for specialised fetal echocardiography owing to suspicion of cardiac pathology increase. For efficient resources allocation, verification of referral indications is required. A knowledge of fetal outcome is important to adequately counsel parents. We aimed to evaluate the diagnoses made, co-morbidity found, and outcome of fetuses referred for echocardiography in a tertiary referral center.

Methods: All prenatal referrals between April 1999 and April 2009 were reviewed. The final cardiac diagnoses, co-morbidity and outcome were recorded.

Results: The cohort included 623 fetuses. Cardiac pathology was found in 30I (48\%), 243 (81\%) had congenital heart defects (CHDs), (arrhythmia in 39 and cardiomyopathy/myocarditis/cardiac tumor in 19). The CHD diagnoses were similar to other series and most fell in the severe spectrum. An increased nuchal translucency (NT) was the main referral indication (32\%). CHD was found in 81/239 (34\%) fetuses with an increased NT. Chromosomal anomalies were found in 26\% (63/243) of the fetuses with CHD. Extra-cardiac anomalies were found in 23\% (4I/ / 80) of the chromosomally normal fetuses with CHD. The mortality rate for CHD with normal chromosomes was 43\% (77/I80).

Overall the termination of pregnancy (TOP) rate for cardiac pathology was 24.2\% (70/289) and for CHD 51\% (I25/243), 61\% (28/46) and $44 \%$ (68//55) when diagnosed before 19 and 24 weeks' gestation respectively.

Conclusions: The referral indications for fetal echocardiography were appropriate as almost half had cardiac pathology. The mortality was high. Fetal outcome and the decision for termination of pregnancy correlated with the CHD severity and presence of co-morbidity.

\section{Accuracy and benefit of prenatal diagnosis of cardiac defects in a referral centre}

\section{Sally-Ann Clur, Peter Mathijs van Brussel, Jaap Ottenkamp and Caterina Madelena Bilardo}

Department of Pediatric Cardiology, Emma Children's Hospital, Academic Medical Centre (AMC) and Centre for Congenital Heart Anomalies Amsterdam-Leiden (CAHAL), The Netherlands

Objective: Good prenatal diagnosis (preDx) of congenital heart defects (CHDs) requires accurate and complete fetal echocardiograms and can reduce postnatal morbidity and mortality. Our aim was to evaluate the accuracy and benefit of prenatal diagnosis in fetuses referred for echocardiography in our unit.

Patients and methods: A retrospective study of fetuses referred for echocardiography between April 1999 and December 2008 was performed. The prenatal and postnatal CHD diagnoses were reviewed and allocated a modified Aristotle (modA) and Wald-score and then compared. The Wald-score at final diagnosis was used to evaluate the benefit of preDx.

Results: Of the 600 included fetuses, 31 I (51.8\%) had a normal heart and 289 (48.2\%) cardiac pathology (CHD in 232 (80.3\%); arrhythmia in 39 ( 13.5\%); cardiomyopathy/myocarditis/cardiac tumour in 18 (6.2\%)). The average prenatal modA-score (4.3 \pm 5.7$)$ correlated well with the postnatal score $(4.1 \pm 5.7)$ (average difference of $0.2 \pm 1.7)$. In 5 I fetuses the postnatal course differed from the prenatal expectation, negatively in 20, (5 potentially critically). PreDx was beneficial in $71.7 \%$ (430/600) of the fetuses. The overall mortality rate was $27.7 \%$ and 50.9\% (1 18/232) for fetuses with CHD. The average modA-score in surviving babies with CHD and normal karyotype was $6.8 \pm 5.1$ and $13.0 \pm 3.3$ in those that died, ( $13.1 \pm 2.9$ in cases of pregnancy termination).

Conclusions: PreDx and counselling for cardiac defects were accurate, beneficial and appropriate, except in cases of suspected aortic coarctation. The mortality was high. The decision to terminate a pregnacy and outcome both correlated with the CHD severity. 
Cardiac function and nuchal translucency in Trisomy 21 fetuses

\section{Sally-Ann Clur, Jaap Ottenkamp and Caterina Madelena Bilardo}

Department of Pediatric Cardiology, Emma Children's Hospital, Academic Medical Centre (AMC) and Centre for Congenital Heart Anomalies Amsterdam-Leiden (CAHAL), The Netherlands

Background: The increased nuchal translucency (NT) is a marker for Trisomy $2 \mathrm{l}$ (Tri2 I) and cardiac defects (CHDs) and has been associated with cardiac dysfunction. We aimed to study cardiac function in Tri2I fetuses.

Methods: Echocardiography was performed on 48 Tri2I fetuses and 190 normal controls, (86 with a normal NT and I04 with a NT=95th percentile), between II and 35 weeks' gestation. Measurements of cardiac function included: E- and A-wave peak velocity, E/A velocity ratio, E/TVI ratio over atrioventricular valves, myocardial performance index, acceleration time (AT), semilunar valve peak velocity (PVel), stroke volume and cardiac output and the ductus venosus pulsatility index for veins at II I 4 weeks' gestation (DVPIV).

Results: I I-I3.9 weeks' gestation: Semilunar valve PVel and tricuspid valve (TV) A-wave velocity were significantly lower and TV E/A ratio and DVPIV significantly higher in Tri2I fetuses. The TV E-wave velocity was significantly lower in Tri2I fetuses without CHD compared to controls with increased NTs.

14 and 2I.9 weeks' gestation: Aortic PVel, mitral valve (MV) E-wave and A-wave velocities, MV E/TVI andTV A-wave velocity were significantly reduced in Tri2I fetuses without CHD compared to controls regardless of NT thickness.

22 and 35 weeks' gestation: A-wave of both ventricles, TV E/TVI and aortic PVel were significantly reduced and pulmonary valve AT significantly increased in Tri2I fetuses.

Conclusions: Cardiac function is altered in Tri2I fetuses, irrespective of the NT thickness or presence of CHD. We found evidence for increased preload in early gestation and diastolic dysfunction of both ventricles with increased afterload thereafter.

\section{Fetal Bradycardia:Tissue Doppler imaging in the diagnosis of long QT syndrome, preliminary report}

Sally-Ann Clur, Merel Bakker, Jaap Ottenkamp, Caterina Bilardo, Irene Kuipers and Dianne de Bruin-Bon

Department of Pediatric Cardiology, Emma Children's Hospital, Academic Medical Centre (AMC) and Centre for Congenital Heart Anomalies Amsterdam-Leiden (CAHAL), The Netherlands

Background: Fetal ECGs are unavailable. Cardiac time intervals can however be measured with Tissue Doppler Imaging (TDI). Fetal-QTc measurement could assist the prenatal diagnosis/exclusion of long QT syndrome(LQTS).

Aim: Measurement of fetal-QTc using TDI.

Methods: TDI was performed on 6 fetuses. TDI-QTc was calculated using Bazett's formula (QTc=QT/vRR) and was/will be compared to the postnatal ECG-QTc.

TDI-QTc validation: TDI-QTc and ECG-QTc measured in mother of case 2 and compared.

Two cases of fetal bradycardia were included:

Case I presented at 22+4 weeks' gestation. No PR prolongation or heart block. Progressive right ventricular dysfunction was seen. Sinus bradycardia or LQTS were considered. Parental ECGs were normal. Maternal SS-A+SS-B antibodies were positive. Fetal TDI-QTC was normal. Postnatally sinus bradycardia and normal QTc was confirmed and cardiac function improved.

Case 2 presented at 27+6 weeks' gestation. Mother: DNA confirmed LQTS II, taking metoprolol. Maternal B-blocker induced fetal bradycardia or fetal LQTS were considered. Amniocentesis was declined. 
Results:

\begin{tabular}{|c|c|c|c|c|c|}
\hline Patient & Gest. age (weeks) & Diagnosis & Heart Rate (b/min) & TDI-QTc (ms) & $\begin{array}{c}\text { Postnatal } \\
\text { ECG-QTc (ms) }\end{array}$ \\
\hline Case I & $29+5$ & $\begin{array}{l}\text { SSA+SSB induced sinus } \\
\text { bradycardia+myocarditis }\end{array}$ & 108 & 410 & 405 \\
\hline \multirow[t]{3}{*}{ Case2 } & $27+6$ & & 133 & 380 & \\
\hline & $32+6$ & B-blocker bradycardia & 134 & 390 & Still in-utero \\
\hline & $36+5$ & & 112 & 429 & \\
\hline Case3 & $32+2$ & Syndromal+SVASD & 144 & 417 & " \\
\hline Case4 & $17+4$ & Twinl & 148 & 426 & " \\
\hline Case5 & $24+1$ & Pericardial effusion & 146 & 353 & " \\
\hline Case6 & $24+1$ & Normal & 126 & 384 & “" \\
\hline
\end{tabular}

Mother of case 2: TDI-QTc=473ms and ECG-QTc469ms, heart rate 67/min.

Conclusions: TDI is possible in the fetus and can be used to reliably measure fetal-QTc.TDI can be helpful in the prenatal diagnosis of LQTS.

\section{Beta-blockers in the management of Congenital Long QT Syndrome}

\section{S.A. Clur, P. Chockalingham, B.M. Beckman, F. van den Heuvel, S. Kaab, N.A. Blom and A.A.M. Wild}

Department of Pediatric Cardiology, Emma Children's Hospital, Academic Medical Centre (AMC) and Centre for Congenital Heart Anomalies Amsterdam-Leiden (CAHAL), The Netherlands

Introduction: Beta-blockers (BB) form the mainstay in managing Congenital Long QT Syndrome (LQTS). Recent findings show that propranolol blocks late non-inactivating sodium currents while metoprolol does not. We aimed to study the electrocardiographic differences among patients using different BB.

Methods: Study population included genotype-positive pediatric LQTS patients ( $\mathrm{n}=79$ ), divided into 3 groups based on initial BB treatment (propranolol $[P]=35$, metoprolol $[M]=31$, atenolol $[A]=13$ ). Subset I included 17 subjects in group $P$ who changed from $P$ to $M$ during follow-up and subset 2 included all 13 subjects in group A who changed from A to M. Manual QTc measurements were made from lead II by an investigator blinded to therapy details.

Results: Gender distribution, type of LQTS and mode of presentation were not significantly different among the 3 groups. Baseline (BL) heart rate and QTc of group P were significantly higher compared to group M (93 \pm 21 vs $79 \pm 2$ lbpm, p=0.0 I, $462 \pm 33$ vs $442 \pm 35 \mathrm{~ms}$, $p=0.02)$. More QTc-shortening was produced by P (462 \pm 33 to $447 \pm 29 \mathrm{~ms}, \mathrm{P}=0.047$, time interval $9 \pm 9$ months) compared to $\mathrm{M}$ (442 \pm 35 to $438 \pm 40 \mathrm{~ms})$ and $\mathrm{A}(460 \pm 29$ to $458 \pm 36 \mathrm{~ms})$. The longer the BL QTc, the more pronounced was the QTc-shortening (mean-SEM, see figure) in group P. The QTc of subset I was significantly shorter prior to change from $P$ to $M(450 \pm 29$ vs $463 \pm 4$, $P=0.038)$, an effect not seen in subset 2 .

Conclusions: Propranolol has a significantly better QTc-shortening effect compared to metoprolol and atenolol and this effect is stronger in patients with longer QTc. Propranolol should probably be first-line therapy in LQTS. 


\section{Is fetal cardiac function gender-dependent?}

\section{Sally-Ann Clur, Katrien Oude Rengerink, Ben Willem Mol, Jaap Ottenkamp and Caterina Madelena Bilardo}

Department of Pediatric Cardiology, Emma Children's Hospital, Academic Medical Centre (AMC) and Centre for Congenital Heart Anomalies Amsterdam-Leiden (CAHAL), The Netherlands

Introduction: An increased nuchal tranclucency (NT) is more common in males. A delay in diastolic cardiac function (DCF) maturation has been proposed as a possible cause for this and also for the reported gender-related differences in the ductus venosus (DV) flow.

Objective: Determination of gender-related differences in fetal cardiac function.

Methods: Prospective, longitudinal study of fetuses referred for echocardiography between Ist October 2003 and Ist April 2009. Three hundred and ten echocardiograms were performed between II and 35 weeks' gestation on 190 karyotypically or phenotypically normal fetuses with a structurally normal heart. The E- and A-wave peak velocity, E/A velocity ratio, E/TVI ratio over the atrioventricular valves, myocardial performance index, acceleration time (AT) and peak velocity over the semilunar valves, stroke volume and cardiac output as well as the DV pulsatility index for veins at II - I4 weeks' gestation, were measured.

Results: The male:female ratio was | 6:74. The tricuspid valve (TV) E/TVI was significantly higher and pulmonary valve AT significantly lower in females compared to males between II-35 weeks' gestation No other significant differences in the measured parameters of cardiac function were found.

Conclusions: The TV E/TVI is increased and the pulmonary valve AT time decreased in female fetuses between II-35 weeks' gestation compared to males. This suggests better right ventricular (RV) relaxation and increased RV afterload in females, independent of NT thickness. Our finding support the hypothesis of delayed RV DCF maturation in males but are unlikely to underlie the higher frequency of increased NTs in male fetuses.

\section{Intima-media thickness and plaque detection in carotid arteries of apparently low risk individuals to guide initiation of preventive treatment}

\section{Anthony John Dalby, Talib Abdoll-Carrim, Belinda Stevens and Roxanne Harito}

Milpark Hospital, Parktown West, Johannesburg, South Africa

Asymptomatic non-diabetic individuals without atherosclerotic disease with low density lipoprotein cholesterol (LDLc) 3.00-5.00 mmol had carotid ultrasound (CU) to measure carotid intima-media thickness ( CIMT) and detect plaque (PL). The CIMT was graded into low, medium or high risk (HR). The presence of PL was graded as HR. These results were related to demographics, traditional risk factors and lipid profile. 149 individuals (median age 62 years) were included: $56 \%$ males, $22.8 \%$ smokers or ex-smokers, $43.0 \%$ with hypertension and $31.5 \%$ with family history of coronary heart disease (CHD). Total cholesterol averaged $5.79 \mathrm{mmol}$, high density lipoprotein $1.45 \mathrm{mmol}$, LDLc $3.76 \mathrm{mmol}$ and triglycerides 1.41 mmol. The average 10 year CHD risk using the Framingham Risk Score (FRS) was 9.8\% (median 8\%; range I-30\%). HR was present in $59 \%$ overall, rising from $23 \%<50$ years to $54 \%$ in those $50-64$ years to $80 \%$ when $>64$ years. $70 \%$ that had a FRS I0-year CHD risk of $>19 \%$ were HR but this group constituted only $24 \%$ of the HR group overall.

$\mathrm{HR}$ on $\mathrm{CU}$ was found in the majority of asymptomatic, non-diabetic patients $>50$ years old without atherosclerotic disease, whose LDLc was 3.0-5.0 mmol. Only $24 \%$ were eligible for preventive therapy according to FRS. A larger prospective study is required to evaluate the usefulness of $\mathrm{CU}$ in risk assessment at various ages. 


\section{BNP fragment level and angiotensinogen M235T and TI 74M gene polymorphism in heart failure patients}

\section{Pop Dana, Zdrenghea Dumitru Tudor, Procopciuc Lucia, Bodisz Gyorgy and Penciu Oana}

University of Medicine and Pharmacy, Cluj-Napoca, Romania

Purpose: To study the association between the individual presence of the M235T and TI74M genotypes and the BNP fragment (8-29) plasmatic level (as a measure of BNP activity), and also, the combined influence of those variants on the plasmatic level of BNP fragment (8-29).

Methods: There were analysed 50 heart failure patients, 31 males, included in NYHA III- IV functional class, $65.76 \pm 6.29$ years. AGT gene polymorphism was detected by polymerase chain reaction, and the plasmatic BNP fragment (8-29) levels was determined using the ELISAmethod.

Results: The mean value of serum BNP fragment levels was $2991.24 \pm 2034.61 \mathrm{fmol} / \mathrm{ml}$. Considering genotype combination of AGT M235T and TI74M polymorphisms, BNP fragment level, for negative MM-negative TT combination (4427.25 \pm 2669.95 fmol/ml), was surprisingly higher $(p<0.05)$ in comparison with heterozygote MT- heterozygote TM combination ( $600.33 \pm 893.77 \mathrm{fmol} / \mathrm{ml})$, heterozygote MT-negative TT combination $(3417.143 \pm 2170 \mathrm{fmol} / \mathrm{ml}$ ), homozygote TT- heterozygote TM combination $(2088.33 \pm 1252.94 \mathrm{fmol} / \mathrm{ml})$ and homozygote TT- negative TT combination (3177.5 $\pm 2490 \mathrm{fmol} / \mathrm{ml}$ ). Analysing each genotype, BNP fragment levels are higher $(p<0.05)$ in patients negative for AGT M235T mutation (4427.25 $\pm 2669.95 \mathrm{fmol} / \mathrm{ml})$, in comparison with the homozygote TT patients $(2677.14 \pm 2062.62 \mathrm{fmol} / \mathrm{ml})$, and the heterozygote MT patients (2285.62 $\pm 1448.45 \mathrm{fmol} / \mathrm{ml}$ ). ForT I 74M genotype, level of BNP fragment in negative TT patients (3348.37 $\pm 2190.70 \mathrm{fmol} / \mathrm{ml})$, was also higher $(p<0.05)$ then in heterozygote TM patients $(1764.2 \pm 1007.18 \mathrm{fmol} / \mathrm{ml})$.

Conclusion: In HF patients there is a relationship between AGT genetic polymorphism and plasmatic BNP fragment level, but paradoxically, the pathogen angiotensinogen gene variants, M235T and TI74M, are associated with decreased serum levels of BNP fragment.

\section{At the HAART of it all}

\section{Murray Davidson}

Charlotte Maxeke Johannesburg Academic Hospital, South Africa

With an estimated national HIV prevalence of $28 \%$ in South Africa (that is an estimated 5.27 million people infected) during the 2007 national prevalence survey $[\mathrm{I}]$ and the still significant incidence of rheumatic heart disease; it is by no surprise then that a number of patients are being presented for cardiac surgery with dual pathology. Historically, HIV positive patients have been denied cardiac surgery because of the fear that cardiopulmonary bypass would further depress an already depressed immune system and further the progression of the disease to acquired immune-deficiency syndrome (AIDS). It is our surmise from both anecdotal experience and the limited body of available evidence (that follows) that cardiac surgery can be performed in patients infected with HIV with a reasonable mortality and morbidity.

We present the results of a prospective review of the outcomes of HIV positive patient's operated upon at the Charlotte Maxeke Johannesburg Academic Hospital for the period of January 2009 to March 2010. In addition, consenting patients were investigated for the effects of cardiopulmonary bypass upon their immune status with respect to CD4 counts, viral load and inflammatory markers. 


\section{A novel blood pressure device accurate for use in adults: Tensoval duo control}

\section{Annemarie de Greeff, Helgadia Klein Smit, Elsabe Springbok and Andrew H. Shennan}

Maternal \& Fetal Research Unit, King's College, London, United Kingdom

Background: Hypertension is a leading cause of cardiovascular and cerebrovascular disease. Accurate blood pressure (BP) measurement is fundamental in identifying those at risk and the accuracy of automated non-invasive BP measurement is continually evolving and improving. The Tensoval duo control is a self-measurement device that uses a novel combination of auscultatory and oscillometric technology. The device previously achieved the accuracy criteria of both the International Protocol (IP) and the British Hypertension Society protocol in a European adult population. We assessed the accuracy of this device in a South African adult population according to the IP of the European Society of Hypertension.

Methods: Ethical approval was obtained and 33 subjects were recruited from Kimberley Hospital Complex (South Africa). Trained observers took 9 sequential same arm BP measurements from each subject, alternating between mercury sphygmomanometery and the device. Data was entered and analysed using Excel (Microsoft Office) according to the IP guidelines.

Results: The Tensoval duo control passed all the phases of the IP and achieved a mean difference (SD) of -3.2(7.2) $\mathrm{mmHg}$ and 2.0(6.0)mmHg for systolic and diastolic pressures respectively. All observer differences were within $4 \mathrm{mmHg}$.

Conclusion: This study confirms the accuracy and recommendation for clinical use of the Tensoval duo control device in an adult population. It is vital to establish the accuracy of automated BP devices through clinical trials that adhere to recognised guidelines such as the IP. Accuracy of this device in special groups such as pregnancy, arrhythmia etc. is yet to be assessed.

\section{Presentation and complications associated with unilateral absent pulmonary artery in two children}

\section{G. Dumani, P.A. Adams and A.M. Cilliers}

Division of Paediatric Cardiology, Department of Paediatrics and Child Health, Chris Hani Baragwanath Hospital, University of the Witwatersrand, Johannesburg, South Africa

We present two children who presented differently and who both were diagnosed with an unilateral absent pulmonary artery. Both children, ages 3 years and 8 years presented with haemoptysis, but the older child also had a continuous murmur due to the presence of a patent ductus arteriosus. Both had a history of recurrent chest infections and were treated in the past for PTB based only on chest X-ray appearance. The 3 -year old child had digital clubbing and low saturations, while the older child had normal saturations. Other signs shared was the clinical appearance of chronic lung disease viz. hyperinflation and Harrison's sulcus.

Echocardiography showed absent right pulmonary artery and patent ductus arteriosus in both children. These findings were confirmed on cardiac catheterisation with measured pulmonary pressures two thirds of systemic pressures and collaterals identified. Both children had ligation of patent ductus arteriosus to reduce the incidence of pulmonary hypertension. Unfortunately the 3-year old child developed pneumonia post-operatively associated with recurrent episodes of haemoptysis and demised.

Unilateral absent pulmonary artery is a rare vascular anomaly and may be isolated or associated with other cardiac anomalies. Symptomatic patients are often misdiagnosed, mistreated or diagnosis at autopsy. Early diagnosis is important for successful treatment and prevention of further deterioration and associated complications. 


\section{Drug secondary prevention in post-menopausal women with ischaemic stroke}

\section{Zdrenghea Dumitru Tudor, Pop Dana, Sitar-taut Adela and Cebanu Mirela}

University of Medicine and Pharmacy, Cluj-Napoca, Romania

Background: It is known that women with stroke are under-treated, including drug treatment. This aspect was less studied with respect to drugs used for secondary prevention.

Methods: In an urban Romanian community, a representative sample of 108 post-menopausal women with ischaemic stroke aged $>55$ years was studied. We considered secondary prevention by drugs. The results were compared with those registered in a similar sample of 84 men aged $>55$ years with ischaemic stroke. The study was carried out using general practitioner's files during 2007 year.

Results: Women mean age $(69.30 \pm 8.90 y)$ was greater in comparison with men $(66.51 \pm 7.93 y . p=0.02)$, but no significant differences were registered between females and males with respect to diabetes mellitus (32.4\% vs 26.2\%), hypertension (98.1\% vs $94 \%$ ), dyslipidemia (46.3\% vs $36.9 \%$ ). According to literature, as preventive drugs, antiplatelet agents and statins were considered. Aspirin was used in $72.2 \%$ of women

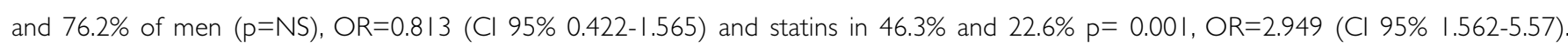
Considering separately the patients under and above 75 years, aspirin was similarly used in women (74.3 vs $67.6 \%$, p =NS) and men (75.7\% vs $78.6 \%, p=N S$ ), but statins were significantly reduced after 75 years, especially in men ( $w-52.7$ vs $32.4 \%, p<0.05 ; m-25.7$ vs $7.1 \%, p<00$ I) Using univariate and multivariate analysis, aspirin use was not significantly influenced by sex, age, cardiovascular risk factors or cardiovascular disease. In turn, statin use was influenced by age, female sex, dyslipidemia and diabetes mellitus.

Conclusion: In post-menopausal women with ischaemic stroke the secondary prevention through aspirin, but mainly through statins is underused, with significant differences in comparison with men in whom the use of statin is even lower.

\section{HDL protects against lethal reperfusion injury via the SAFE pathway}

\section{Miguel Frias, Sarin Somers, Lydia Lacerda, Richard James and Sandrine Lecour}

Hatter Cardiovascular Research Institute, Faculty of Health Sciences, University of Cape Town, South Africa

Background: Recent studies suggest a cardioprotective effect of high-density lipoprotein (HDL) independent from its cholesterol transport activity, but the mechanisms remain unclear. The SAFE (Survivor and Activating Factor Enhancement) is a new potent prosurvival signaling pathway in cardioprotection that involves the cytokine tumour necrosis factor alpha (TNF $\alpha$ ) and the transcription factor Signal transducer and activator of transcription 3 (Stat3). We propose that HDL, given at the onset of reperfusion, confers protection against the lethal reperfusion injury and that this protection occurs via the SAFE pathway.

Methods and results: Langendorff retrograde perfusion was performed on isolated hearts from wild type (WT), TNF $\alpha$-deficient (TNF-KO) and specific-cardiomyocytes Stat3-deficient (Stat3-KO) mice. The hearts were submitted to 35 min global ischaemia followed by 45 min of reperfusion. HDL was given during 7 first min of reperfusion. Infarct size was assessed as an endpoint by triphenyltetrazolium chloride (TTC) staining at the end of the protocol. In control hearts, the infarct size was $41.3 \pm 1.7 \%$. Addition of $\mathrm{HDL}(100,200,400 \mathrm{mg} / \mathrm{ml})$ reduced the infarct size to $33.3 \pm 1.7 \%(p<0.05$ versus control), $25.8 \pm 1.9 \%(p<0.0$ I versus control), $23.3 \pm 2.1 \%(p<0.0$ I versus control), respectively. The protective effect of HDL $200 \mathrm{mg} / \mathrm{ml}$ was abolished in TNF $\alpha-K O$ and Stat3-KO compared to control (HDL in TNF $\alpha-W T: 25.8 \pm 2.9 \%$, TNF $\alpha-K O: 41.6$ $\pm 2.2 \%$; HDL in Stat3-WT $23.3 \pm 2 \%$, Stat3-KO $37.2 \pm 2.2 \%, \mathrm{p}<0.0$ I versus WT).

Conclusions: $\mathrm{HDL}$, given at the onset of reperfusion, induces cardioprotection against lethal reperfusion injury and activation of the SAFE pathway is crucial for this cardioprotective effect. This study underlines the therapeutic potential HDL to confront particular clinical situations. 
Time to fibrinolytics for acute myocardial infarction and reasons for delays at Steve Biko Academic Hospital

\section{R. Meel and R. Gonçalves}

Department of Internal Medicine, Steve Biko Academic Hospital and University of Pretoria, South Africa

Background: Fibrinolytic therapy is a time critical intervention that is proven to reduce mortality and morbidity in patients with ST elevation myocardial infarction (STEMI). Little data is available in South Africa regarding time to fibrinolytic therapy for STEMI patients and reasons for delayed therapy.

Objectives: This study establishes the proportion of STEMI patients receiving fibrinolytic agents at Steve Biko Academic Hospital (SBAH). Furthermore, the time taken between the onset of symptoms and the administration of fibrinolytic therapy was defined. The delays due to patient factors, transfer to hospital and in-hospital delays, and the reasons for those delays, were also established. The impact of the delays was then quantified.

Methods: A prospective, observational study, including 100 patients, was performed. The times between symptom onset to receipt of fibrinolytic therapy and the reasons for delays were documented. This information was collected from patient interviews (following informed consent) and from hospital records.

Results: Only $37 \%$ of patients received fibrinolytic therapy and only $3 \%$ received the medication within one hour. The median total delay in receiving fibrinolytic therapy was 270 minutes (45-584) or 4.5 hours (0.8-9.8). The median time delays from onset of symptoms to call for help, between calling for help and arriving at hospital, and from hospital arrival to fibrinolytic agent administration, were 35 (5-। I85), 55 (12.5-670) and 62.5 (16.5-282) minutes, respectively. Numerous delays were identified at all stages, with patient and transport delays being most significant. Using published data, it was calculated that an additional 32 patients/I 000 treated could have been saved, had the treatment been administered within the first hour.

Conclusions: Although patient and transport factors played the leading role in delayed therapy, in-hospital delays were unacceptably long. Of greater concern was the extremely low rate of fibrinolytic administration at presenting hospitals. Corrective measures are required to rectify this situation.

\section{Sphingosine-I-phosphate (SIP) mediates cardioprotection by modulation of the mitochondrial function}

\section{Damian Hacking, Roisin Kelly, Derek Yellon, Lionel Opie, Derek Hausenloy and Sandrine Lecour}

Hatter Cardiovascular Research Institute, Faculty of Health Sciences, University of Cape Town, South Africa

Background: Sphingosine-I-phosphate(SIP), a major component of the high density lipoprotein (HDL), protects against ischaemia/reperfusion injury. However, the signalling pathways involved in SIP-induced cardioprotection remain unclear. Inhibition of mitochondrial permeability transition pore (mPTP) opening can promote survival against reperfusion injury. Therefore, we hypothesised that SIP may improve the function of cardiac mitochondria and that this will be mediated via modulation of the mPTP pore.

Materials and methods: Isolated male Long Evans rat hearts were perfused on the Langendorff system. After 30 min of stabilisation, 10 min treatment with SIP (IOnM) and 7 min washout period, the heart was removed and mitochondria were isolated using the Percoll method. State 3 and State 4 respiration indices were recorded using a Clarke-electrode system. In addition, adult mouse cardiomyocytes isolated from c57bl6 male mice were treated with SIP (I0 nM) for $30 \mathrm{~min}$ and a $30 \mathrm{~min}$ washout period prior to a 2 hour anoxic stress. Cell viability was measured using Trypan Blue staining. Cells were also stained with TMRM to test mPTP opening.

Results: Pre-treatment with SIP improved the respiratory control index in isolated mitochondria from $4.2+/-X$ to $\mid I .1+/-X(n=4 ; p<0.05)$. Additionally, treatment with SIP significantly inhibited the opening of the MPTP in isolated cardiomyocytes, and increased cell viability from $35.2 \%$ to $50.3 \%(p<0.05)$.

Conclusion: SIP improves mitochondrial function and this effect may be mediated by modulation of mPTP opening. 


\title{
Stellium First-In-Man - 2 year results
}

\section{Farrel Hellig and Damian Conway}

Sunninghill Hospital, Johannesburg, South Africa

\begin{abstract}
A First-In-Man multi-centre clinical study of a low-dose paclitaxel drug eluting stent (PES) was undertaken at 7 hospitals in South Africa in order to investigate provisional safety and efficacy indicators of a low-lose PES, erodible polymer DES strategy in humans. An angiographic (QCA) and optical coherence tomography (OCT) sub-study was conducted at 6 months (previously reported) as well as 12 month and 24 month clinical follow-up. Thirty seven symptomatic patients with angina (CCS functional Class 2-4) but negative Troponin $T$ were recruited and treated with one or more Stellium (DISA Vascular, Pty Ltd) stents. Device success was $98 \%$.
\end{abstract}

Results: 2/43 lesions (4.7\%) required target lesion revascularisations (TLR) and 3/36 patients (8.3\%) suffered MACE at 12 months. There we no deaths at 12 month follow-up. However, shortly after the 12 month clinical follow-up and discontinuation of clopidogrel, one patient died of definite stent thrombosis (confirmed by post-mortem histopathology). There was one further non-device-relatedTLR between 12 and 24 months.

Conclusions: Despite 6 month angiographic and OCT evidence and two-year clinical evidence of promising efficacy of the Stellium stent, the single confirmed very late stent thrombosis (with histological evidence of ongoing drug activity) suggests that while a very low dose of paclitaxel retains the capacity of a much larger dose PES to attenuate clinically significant neointimal hyperplasia, the risk of delayed healing and late thrombosis also remains. This occurs despite the fact that the polymer carrier is fully erodible and hence the presumed contributing factor of long-term drug leaching is negated.

\section{From bench to bedside: Correlation of mutation and clinical diagnosis in progressive familial heart block type I (PFHBI)}

\section{Lou Hofmeyr*, Althea Goosen*, Carin Green", Valerie Corfield" and Paul Brink*}

*Department of Internal Medicine, Faculty of Health Sciences, University of Stellenbosch, South Africa

\#Department of Biomedical Science, Faculty of Health Sciences, University of Stellenbosch, South Africa

$\mathrm{PFHBI}$ is an autosomal dominantly inherited conduction disorder causing right bundle branch block (RBBB), bifasicular block (BfB) and complete heart block (CHB). Earlier publications included I st degree AV block ( $\left.{ }^{\circ} \mathrm{AVB}\right)$, anterior or posterior hemi-block and incomplete RBBB (IRBBB) as part of the spectrum of disease. The high incidence of BBB and CHB in the general population make the false diagnosis of an inherited heart block possible. Mutational and functional analysis of genes on chromosome 19q13 has identified the c.19G-->A mutation in the transient receptor potential channel (TRPM4) gene as the cause of PFHBI. The mutation-associated phenotype could thus be studied. Within families segregating PFHBI 158 individuals were investigated. Of 77 regarded as affected II (I4.3\%) were non-mutation carriers (NMC). The NMC group included I patient with a permanent pacemaker, I patient with RBBB or BfB, I with $1^{\circ} \mathrm{HB}, 2$ with hemi-block and 2 with IRRB. Of the 8 I patients thought to be unaffected, 6 (7.4\%) were mutation carriers (MC). This means that 17 (9.2\%) patients have been incorrectly assessed in the past.

Identification of the mutation allowed unequivocal identification of at-risk individuals by DNA-based diagnosis. The typical ECG features of $\mathrm{PFHBI}$ are RBBB and BFB progressing to CHB. Other ECG abnormalities were incorrectly included in the phenotype. This is proof of genomic medicine moving from bench to bedside in the evaluation and management of patients with PFHBI. 


\section{An unusual case of quadrivalvar heart disease in an I I-year old male child}

\section{Ebrahim Goolam Mahomed Hoosen, J.J. Patel, Sajidah Khan and Andiswa Nzimela}

Paediatric Cardiology, Inkosi Albert Luthuli Central Hospital, Durban, KwaZulu-Natal, South Africa

An II-year old male child was referred with Grade III functional disability (NYHA). This had begun a year before presentation with progressive dyspnoea and oedema. No recent febrile illness, arthritis, arthralgia or other clinical manifestations of acute rheumatic fever where ever noted previously.

He was found on echocardiography to have stenoses of the mitral, aortic, tricuspid and pulmonary valves. Mild or trivial regurgitation of the mitral, pulmonary and tricuspid valves where recorded with moderate aortic regurgitation. The echocardiographic appearance of the mitral and aortic valves in particular where consistent with Rheumatic Heart Disease (RHD). No evidence of connective tissue or infiltrative disorders where identified.

He was managed by initial balloon dilatation of the aortic valve. This was followed by balloon dilatation of his mitral, pulmonary and tricuspid valves at a second sitting. He made an excellent recovery with significant reductions in gradients across all 4 valves and a marked improvement in exercise tolerance.

Few cases of quadrivalvar heart disease have been reported with involvement of the pulmonary valve particularly uncommon. All of these have been in adults with all attributed to RHD. To the best of our knowledge, this is the first reported case in a patient of this age.

\section{The cardioprotective effects of Prosopus glandulosa}

\section{Barbara Huisamen, Cindy Hill, Daneel Dietrich and Amanda Lochner}

Division of Medical Physiology, Department of Biomedical Sciences, Faculty of Health Sciences, University of Stellenbosch, South Africa

The dried, pulverised pods of P. glandulosa are currently marketed in South Africa as an over-the-counter food supplement with anti-hypertensive properties. These allegations were tested using rat and mice models of obesity, insulin resistance and hypertension.

Wistars rats were placed on either a high caloric (HC) or high fat (HF) diet to induce obesity/insulin resistance or obesity/hypertension respectively and compared to age-matched controls. Half of each group was treated with the plant material set in jelly blocks from the onset of the change in diet or from week 8 of a 16 week diet period. In addition, conditional cardiac insulin receptor $\mathrm{KO}$ mice (CIRKO) were treated for an eight week period. Blood pressure of the HF animals was monitored on a weekly basis using a tail cuff. At the end of the treatment period, animals were sacrificed and hearts perfused ex vivo to determine infarct size after 35min regional ischaemia, 120min reperfusion. Infarct size in mouse hearts was determined after 20 min global ischaemia, 60 min reperfusion. Ventricular cardiomyocytes were prepared by standard methods and insulin sensitivity tested via deoxyglucose accumulation.

Results showed a reduction in infarct development in all untreated vs treated groups ( $n=9$ ): control: $45.1 \pm 2.6 / 36.8 \pm 3.1, P<0.05 ; \mathrm{HC}: 43.1$ $\pm 3.7 / 36.4 \pm 3.1, \mathrm{P}<0.05$; C57BL6: $43 \pm 3.4 / 27 \pm 2.7, \mathrm{P}<0.0 \mathrm{I}$; CIRKO: $49.5 \pm 3.6 / 28.1 \pm 3 \mathrm{P}<0.00 \mathrm{I}$. In the HF group, treatment resulted in both prevention and reversal of hypertension development. Cardiomyocytes were rendered more insulin sensitive.

We conclude that the material obtained from the P. glandulosa plant has both anti-hypertensive and cardioprotective effects. 


\title{
Use of vascular plugs in pulmonary arteriovenous malformation (AVM) - not always possible
}

\section{J.A.Joshi, F.F.Takawira and B.J.Mitchell}

Department of Paediatric Cardiology, Steve Biko Academic Hospital and University of Pretoria, South Africa

Introduction: Two children with different forms of pulmonary arteriovenous malformations (AVM) are discussed with regards to their differing aetiologies and management outcomes.

Case presentation: A 2-year old and 5-year old both presented with similar histories of decreased effort tolerance. Both children were noted to have digital clubbing and cyanosis with oxygen saturations in the low 70s. The chest $X$-ray of the first child showed a diffuse interstitial pattern while that of the second child showed a dilated left atrium with an opacity over the right lower lobe. Echocardiograms were performed on both children and revealed significant left heart dilatation. Cardiac anatomy and function were normal. Contrast echocardiograms were then performed and were positive indicating the presence of extra cardiac left to right shunts. Selective RPA angiography of the first child demonstrated a large AVM. A $16 \mathrm{~mm}$ Amplatzer vascular plug II was placed in the feeding vessel and successfully occluded the AVM. Cardiac angiography of the second child demonstrated the presence of bilateral diffuse AVMs. A computer tomographic scan of the brain confirmed the presence of a large vein of Galen AVM. With the finding of multiple AVMs a clinical diagnosis of Rendu-Osler-Weber syndrome or hereditary haemorrhagic telengiectasia $(\mathrm{HHT})$ was made. Due to the diffuse nature of the AVMs further surgical or transcatheter treatment was not deemed possible.

Discussion: Approximately 70\% of congenital pulmonary AVMs are associated with Rendu-Osler-Weber syndrome of hereditary haemorrhagic telangiectasia. The rest may be idiopathic or the acquired forms may develop after Glenn shunts or modified Fontan procedures. Pulmonary AVMs form a spectrum anatomical abnormalities. Treatment options vary from transcather closure of feeder vessels using coils or vascular occlusive devices to surgery.

\section{Ethanolamine: A novel mediator of cardioprotection}

\author{
Roisin Kelly*, Kim T. Lamont", Jonathan C. King", Sarin Somers", Damian Hacking", Lionel H. Opie" and \\ Sandrine Lecour\# \\ *Department of Human Biology, University of Cape Town, South Africa \\ \#Hatter Cardiovascular Research Institute, Faculty of Health Sciences, University of Cape Town, South Africa
}

Aims: Sphingosine-I-phosphate (SIP) protects the heart against ischaemia-reperfusion (I/R) injury. There is indirect evidence in the literature suggesting that SIP can form ethanolamine (Etn) in the presence of fatty acid amide hydrolase (FAAH). Also, signal transducer and activator of transcription 3 (STAT-3) is a critical prosurvival factor in I/R injury. Therefore, we propose that SIP protects the heart by stimulating the production of ethanolamine and activation of STAT-3.

Methods: In vivo rat hearts were subjected to $30 \mathrm{~min}$ ischaemia/2 h reperfusion is the presence or absence of $5 \mathrm{mg} / \mathrm{kg} \mathrm{SIP}$ and/or the FAAH inhibitor, URB597. Isolated hearts from wild type or cardiac specific STAT-3 knockout (K/O) mice were pretreated with SIP (I0 nmol/L) or Etn $(0.3 \mathrm{mmol} / \mathrm{L})$ before an I/R insult. Infarct size was measured at the end of each protocol by triphenyltetrazolium chloride staining.

Results: SIP decreased infarct size in rats subjected to I/R in vivo (Control; $6 I \pm 3 \%$ vs. SIP; $43 \pm 4 \%, p=0.0$ I). However, this protection was inhibited by URB597. Pre-treatment with SIP or Etn decreased phosphorylated cytosolic STAT-3 [control; $0.87 \pm 0.19$ vs. Etn; $0.28 \pm 0.12$ arbitrary units or control; $1.4 \pm 0.1$ vs. SIP; $0.6 \pm 0.1$ arbitrary units, $p<0.05$ vs. control] and decreased infarct size in both isolated rat and mouse hearts after I/R. However, this protective effect was lost in cardiomyocyte specific STAT-3 K/O mouse hearts.

Conclusion: Our findings delineate a novel protective signalling pathway in which SIP can confer protection against I/R injury via Etn induced activation of STAT-3. 


\section{A successful primary angioplasty programme in a low volume private sector South African hospital}

\section{David Kettles}

East London Private Hospital, Vincent, East London, South Africa

Introduction: Percutaneous coronary intervention (PCI) is preferred reperfusion therapy for acute ST-elevation myocardial infarction (STEMI). South African application of this technique is unpublished. Low volume units may not duplicate published outcomes. East London Private Hospital has run a 24-hour primary angioplasty unit since 2000, with 2 attending cardiologists, I experienced sister, I radiographer and 2 cardiac technologists in attendance. We audited our practice between 2006 and 2009.

Methods: Demographic, initial clinical and procedural data was obtained prospectively from consecutive STEMI patients presenting to our catheterisation laboratory. Additional data was collected at discharge and initial follow-up visit.

Results: 126 patients had emergency angiography for presumed STEMI between September 2006 and December 2009: 79\% male, median age 54 years. Diabetes was present in 24\%, hypertension in 53\%, and $42 \%$ were current smokers. Patients presented with median symptom onset to hospital admission time of 120 minutes: 47\% presented within 2 hours, 92\% within six hours. 29\% had anterior infarction. I I 8 patients had PCl: 92\% achieved TIMI3 flow post procedure. 72 drug eluting stents were implanted, 73 bare metal stents: angioplasty only in 5 patients. Door to balloon time (dtbt) was median 72 minutes: $83 \%$ of patients were treated within 90 minutes. Patients presenting initially to the PCl hospital had median dtbt of 51 minutes, 83\% were treated within 90 minutes. In hospital mortality was 1.6\%, 30 day mortality $2.4 \%$.

Conclusion: This low volume interventional unit achieves procedural results and patient outcomes comparable with the published literature from high volume academic centres.

\section{The CI-C2 region of cardiac myosin binding protein C interacts with cardiac Troponin I}

\section{Craig Kinnear, Amsha Ramburan, Lundi Korkie, Johann Riedemann and Johanna Moolman-Smook}

US/MRC Centre for Molecular and Cellular Biology, Department of Biomedical Sciences, University of Stellenbosch, South Africa

Cardiac myosin binding protein C (cMyBPC) is a large multidomain protein and is an essential component of the thick filament of the sarcomere. The quaternary structure of this protein still remains uncertain and its function has not yet fully been characterised. Nonetheless, it has been proposed to constrain cardiac contractility by placing an internal load on the cross-bridge cycle. This effect is, however, not brought about via tethering of myosin heads to the thick filament and has been proposed to involve interaction between the $\mathrm{N}$-terminal regions of $\mathrm{CMyBPC}$ and the thin filament. In order to further investigate this mechanism, we sought to identify novel interactors of the cMyBPC $\mathrm{Cl}-\mathrm{C} 2$ region of MyBPC. Using yeast two-hybrid library screening, followed by in vitro and in vivo co-immunoprecipitation, bioluminescent resonance energy transfer and three-dimentional colocalisation, we identified cardiac troponin I (cTNI) as a prominent cMyBPC-interacting protein. Furthermore, we showed that the interaction occurs preferentially when the MyBPC motif is phosphorylated. Our data therefore provide new insights into mechanisms by which cMyBPC affects cross-bridge cycling. 


\title{
Percutaneous pericardioscopy and pericardial biopsy in suspected tuberculous pericarditis: Avoiding unnecessary anti-tuberculous therapy
}

\section{Charles Kyriakakis, Helmuth Weich and Anton Doubell}

Division of Cardiology, Department of Medicine, University of Stellenbosch and Tygerberg Hospital, South Africa

\begin{abstract}
Background: The high incidence of mycobacterium tuberculosis as a cause of pericardial effusion in sub-Saharan Africa often leads to empiric anti-tuberculous therapy. We have previously demonstrated, in a pilot study of II patients, that in suspected cases of tuberculous (TB) pericarditis the use of percutaneous pericardioscopy and pericardial biopsy allows for a more rapid diagnosis of TB pericarditis via the demonstration of Acid Fast Bacilli (AFB's), compared to pericardial fluid evaluation which is culture dependant (median time 22 days). The pilot group has been expanded to 18 patients and we now demonstrate the value in excluding pericardial TB via biopsy in avoiding unnecessary exposure to anti-tuberculous therapy.
\end{abstract}

Method: Patients presenting with a large non-traumatic pericardial effusion (epicardial separation distance $>10 \mathrm{~mm}$ ) were offered participation. Each underwent pericardiocentesis via a standard procedure followed by percutaneous pericardioscopy and pericardial biopsy. Pericardial fluid evaluation included: biochemistry, adenosine deaminase level, cell count, AFB's and TB culture. Pericardial biopsy evaluation included: AFB's, TB culture and histology for granulomas.

Results: Nine (50\%) of 18 patients were found to have definite pericardial TB, all biopsy proven. An alternative cause for the pericardial effusion could be identified in all the remaining patients. These included: one carcinomatous effusion; a systemic lupus erythematosis serositis; a bacterial pericardial empyema; a delayed collection due to prior chest trauma; and four were classified as idiopathic pericardial effusions.

Conclusion: The evaluation of suspected pericardial tuberculosis via pericardioscopy and pericardial biopsy ensures that patients with an alternative cause for the effusion are not exposed to anti-tuberculous therapy.

\section{Does obesity and/or diabetes influence the outcome of ischaemic post-conditioning?}

\section{Lydia Lacerda*, Lionel Opie" and Sandrine Lecour"}

"Department of Human Biology, University of Cape Town, South Africa

\#Hatter Cardiovascular Research Institute, Faculty of Health Sciences, University of Cape Town, South Africa

Introduction: Ischaemic post-conditioning is an additional powerful tool for reducing infarct size in patients with myocardial infarction. However, successful clinical studies have not considered obese and/or diabetic patients. We, therefore, aimed to investigate the effectiveness of IPostC in obese or diabetic mice.

Methods: A high carbohydrate diet was fed to male mice for I I weeks. Diabetes was induced with a single streptozotocin (STZ) injection I0 days prior to the experiment. Mice were tested for body weight, glucose, insulin and leptin. Isolated hearts were subjected to an ischaemiareperfusion (I/R) protocol of $35 \mathrm{~min}$ ischaemia followed by $45 \mathrm{~min}$ reperfusion. IPostC was initiated at onset of reperfusion with $6 \times 10$ sec cycles of alternating reperfusion/ischaemia.

Results: HCD increased weight from $29.0 \pm 1.3$ to $32 \pm 0.7$ grams, $p<0.05$. Plasma insulin and leptin were increased from $0.46 \pm 0.03$ to $0.64 \pm 0.06 \mathrm{ng} / \mathrm{ml}, \mathrm{p}<0.05$ and from $4.5 \pm 0.4$ to $10.6 \pm 0.9 \mathrm{ng} / \mathrm{ml}, \mathrm{p}<0.00 \mathrm{l}$, respectively, while glucose was unchanged. In STZ-treated mice the body weight and insulin levels remained unchanged. Leptin was significantly reduced from $4.5 \pm 0.7$ (CTL) to $0.6 \pm 0.2 \mathrm{ng} / \mathrm{ml}, \mathrm{p}<0.0 \mathrm{l}$. Similar to control animals, IPostC reduced infarct size by $31.5 \%$ in obese mice and by $20.4 \%$ in STZ-treated mice.

Conclusion: Both obese and diabetic mice can be protected with ischaemic post-conditioning. Our data suggest that Type I diabetic and/or modestly obese patients may still benefit from IPostC. 


\section{Drinking red wine: a SAFE sip away from cardioprotection}

\section{Kim Lamont, Sarin Somers, Lionel Opie and Sandrine Lecour}

Hatter Cardiovascular Research Institute, Faculty of Health Sciences, University of Cape Town, South Africa

Aim: Epidemiological studies suggest that moderate chronic consumption of red wine confers cardioprotection but the mechanism remain unclear. We propose that both melatonin and resveratrol, as found in red wine, protect via the activation of the newly discovered prosurvival SAFE (Survivor Activating Factor Enhancement) pathway that involves the activation of TNF and the transcription factor STAT3.

Methods: Isolated perfused mouse (wildtype, TNF receptor 2 knockout mice, cardiac specific STAT3 deficient mice) or rat hearts were subjected to ischaemia-reperfusion. Resveratrol $(7 \mathrm{mg} / \mathrm{L})$ or melatonin $(75 \mathrm{ng} / \mathrm{L})$, were perfused for $15 \mathrm{~min}$ with a 10 min washout period prior to an ischaemia-reperfusion insult. Infarct size was measured at the end of the protocol and Western blot analysis was performed prior to the ischaemic insult.

Results: Both resveratrol and melatonin, at concentration found in red wine, significantly reduce infarct size compared with control hearts ( $24 \pm 2 \%$ and $25 \pm 2 \%$, respectively, vs $69 \pm 3 \%$ for control, $\mathrm{p}<0.00 \mathrm{I}$ ) in wildtype mice hearts but failed to protect in TNFR2 knockout or STAT3 deficient mice. Furthermore, perfusion with either melatonin or resveratrol increase STAT3 phosphorylation by $79 \%$ and $50 \%$, respectively, prior to ischaemia ( $p<0.05$ vs control).

Concluclusion: Our data support the red wine hypothesis that melatonin and resveratrol in red wine protect the heart against ischaemiareperfusion via the SAFE pathway.

\section{Prevalence of residual left ventricular structural changes after one year of anti- hypertensive treatment in patients of African descent}

\section{Elena N. Libhaber, Gavin R. Norton, Carlos D. Libhaber, Angela J. Woodiwiss, Geoffrey P. Candy, Mohammed R. Essop and Pinhas Sareli}

Division of Cardiology, Chris Hani Baragwanath Hospital, University of the Witwatersrand, Johannesburg, South Africa

Background: One year of anti-hypertensive therapy may normalise left ventricular (LV) structure in $51 \%$ of hypertensive patients of European descent. Whether similar effects can be achieved in groups of African descent with a high prevalence of concentric LV hypertrophy (LVH) and remodelling is unknown.

Methods: In 103 patients in the Baragwanath Hypertension Study we evaluated the prevalence of concentric LVH and remodelling before and after 13 months of stepwise anti-hypertensive therapy. Left ventricular structure was evaluated with echocardiography and BP control was evaluated with both clinic and ambulatory BP monitoring.

Results: After 13 months of therapy 24-hour BP control was achieved in 47\% of patients and clinic BP control in 64\% of patients. Four months of therapy reduced LV mass index (LVMI, (g/m2.7)) and LV relative wall thickness (RWT) from $63.7 \pm 19.0$ to $53.7 \pm 13.9$ ( $p<0.000 \mathrm{I}$ ), and $0.50 \pm 0 \mathrm{II}$ to $0.47 \pm 0.10$ respectively; a change which persisted until I 3 months in both parameters. Despite these changes in LVMI and RWT, the proportion of patients with a normal LV mass or geometry increased only from 17 to $26 \%$, whilst $25 \%$ remained with concentric $\mathrm{LVH}, 25 \%$ with eccentric LVH and 23\% with concentric LV remodelling. The factors that were independently associated with a residual abnormal LV structure were baseline and final body mass index $(p<0.05)$ or baseline 24-hour systolic BP $(p<0.05)$.

Conclusions: Even after a year of anti-hypertensive therapy a high proportion (74\%) of hypertensives of African ancestry retain residual LV structural changes, an effect that is associated with body size and baseline 24-hour BP. 


\title{
Contribution of central and general adiposity to an abnormal left ventricular diastolic function in a community sample with a high prevalence of obesity
}

\author{
Carlos David Libhaber, Gavin R. Norton, Olebogeng H.I. Majane, Elena Libhaber, Mohammed R. Essop, \\ Richard Brooksbank, Muzi Maseko and Angela J. Woodiwiss
}

Division of Cardiology, School of Physiology, Chris Hani Baragwanath Hospital, University of the Witwatersrand, Johannesburg, South Africa

The relative independent contribution of an excess adiposity as indexed by measures of central, general or peripheral adiposity toward an abnormal cardiac diastolic chamber function at a community level is unclear. In 377 randomly selected participants over 16 years of age from a community sample with a high prevalence of excess adiposity ( $\sim 25 \%$ overweight and $\sim 43 \%$ obese) we assessed the independent contribution of indexes of adiposity to the variation in E/A. After adjustments for a number of confounders including age, gender, pulse rate, conventional diastolic (or systolic) BP, anti-hypertensive treatment, left ventricular mass index and the presence of diabetes mellitus or an $\mathrm{HbAIc}>6.1 \%$; waist circumference was an independent predictor of a reduced $E / A(p=0.0038)$. In contrast, neither body mass index ( $P=0.07)$, waistto-hip ratio $(p=0.23)$ nor skin-fold thickness $(p=0.37)$ were independently associated with $E / A$, and waist circumference was independently associated with $E / A$ even after adjustments for other adiposity indexes including body mass index $(p<0.05-0.005)$. In contrast to the effects on diastolic function, waist circumference was not correlated with left ventricular ejection fraction $(p=0.23)$. The independent relationship between waist circumference and $E / A$ (standardised $B$-coefficient $=-0.14 \pm 0.05, p=0.0038$ ) was second only to age (standardised $B$-coefficient $=-0.57 \pm$ $0.05, p<0.000 \mathrm{I}$ ) and similar to blood pressure (standardised $\beta$-coefficient $=-0.11 \pm 0.04, p=0.0075$ ) in the magnitude of the independent effect on E/A. The inclusion of relative wall thickness rather than LVMI in the regression equation produced similar outcomes. The exclusion of left ventricular mass index and relative wall thickness from the regression equations, or the inclusion of carotid-femoral pulse wave velocity, or 24-hour BP as confounders, failed to modify the relationship between waist circumference and E/A. In conclusion, waist circumference is second only to age in the impact of the independent association with E/A in a population sample with a high prevalence of excess adiposity. This effect was not accounted for by left ventricular hypertrophy or remodeling, 24-hour BP or arterial stiffness.

\section{Melatonin-induced cardioprotection is dependent on both STAT3 and ERKp44/p42 activation}

\section{A. Lochner, F. Nduhirabandi, S. Genade and A. Genis}

Department of Biomedical Sciences, University of Stellenbosch, South Africa

Melatonin, the hormone secreted by the pineal gland, confers significant protection against myocardial ischaemia/reperfusion damage. We previously demonstrated that these actions were melatonin receptor dependent. The aim of this study was to determine the significance of STAT3 and the RISK pathway as putative downstream effectors.

Methods: Rats received melatonin orally or intraperitoneally $(5 \mathrm{mg} / \mathrm{kg})$ daily for 16 weeks or 10 days respectively. After removal, perfused hearts were subjected to $35 \mathrm{~min}$ regional ischaemia/2 $\mathrm{h}$ reperfusion for determination of infarct size. In another series, melatonin $(50 \mu \mathrm{M})$ was administered for $10 \mathrm{~min}$ with or without washout before and after 20 min global ischaemia, +/- AG490 (I00 nM, STAT inhibitor) or

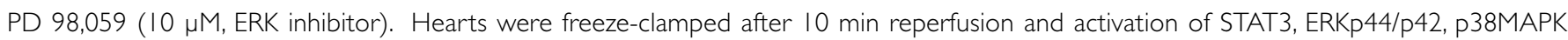
and PKB/Akt was evaluated by Western blot.

Results: Melatonin, regardless of its mode of administration, causes a significant reduction in infarct size: for example, controls: $41.29 \pm 2.94$; melatonin pretreatment for 10 days: $26.09 \pm 3.13, p<0.01$ ). Melatonin pretreatment followed by washout caused activation of STAT3 before and after sustained ischaemia. Melatonin pretreatment without washout caused a significant activation of ERK $p 44 / p 42$ during early reperfusion, but no change in PKB/Akt or STAT3. Co-perfusion of melatonin with AG490 or PD98,059 caused abolishment of cardioprotection, as indicated by increases in infarct size (melatonin + AG490: $49.07 \pm 2.65$; melatonin + PD: $43.62 \pm 5.62 ; \mathrm{p}>0.05$ vs melatonin pretreatment).

Conclusions: Melatonin-induced activation of STAT3 and ERKp44/p42 depends on the protocol, but both play pivotal roles in cardioprotection. 


\section{Sinus of Valsalva aneurysm and left ventricular non compaction: Is there a link?}

\section{William Wayne Lubbe, Anton Doubell and Philip Herbst}

Division of Cardiology, Department of Medicine, University of Stellenbosch and Tygerberg Hospital, South Africa

We present echocardiographic images of a young man referred to our cardiac clinic. He had complained of an intermittent cough and NYHA Class II dyspnoea. The referral hospital had detected cardiomegaly on a chest radiograph and an echocardiogram demonstrated a "cystic mass" in his anterior mitral valve leaflet (AmVL).

Repeat echocardiography revealed a sinus of Valsalva aneurysm (SVA) of the left coronary cusp dissecting into the AMVL with thrombus lining the aneurysm. This was associated with mild mitral and aortic valve regurgitation. Surprisingly, the left ventricle was also dilated and noncompacted (LVNC) with moderately impaired systolic function.

SVA involving the left coronary cusp is rare. To our knowledge this is the first described case of a SVA dissecting into the AMVL. No clear mechanistic association between sinus of Valsalva aneurysm and LVNC has hitherto been described. A single case report exists which describes LVNC in a patient with a SVA involving the right coronary cusp. One further case report describes this combination of defects in a patient with associated congenital heart disease.

Several disease loci have been identified in humans that may be responsible for LVNC but these loci are not associated with aortic root pathologies. A more recent publication shows that in mice, reduced versican cleavage due to Adamts9 haploinsufficiency is associated with aortic root abnormalities and "spongy" myocardium consistent with non-compaction of the left ventricle. This potential link has not been investigated in humans.

\section{Utility of 3-dimensional transoesophageal echocardiography:A single centre experience}

\section{F. Mamdoo, F. Peters, H. Matioda and M.R. Essop}

Division of Cardiology, Chris Hani Baragwanath Hospital, University of the Witwatersrand, Johannesburg, South Africa

Background: Three dimensional transoesophageal echocardiography(3DTEE) represents a relatively new imaging modality.

Aims: To evaluate the utility of 3DTEE in routine clinical practice and to compare this modality against standard two dimensional echocardiography (2DTEE).

Methods: 3DTEE using a matrix array transducer (X7-2) coupled to a Philips iE33 system was performed in 87 consecutive patients from January 2010 to May 2010. 3DTEE was performed in all patients under conscious sedation with midazolam. All images were recorded on an Excelera system (Philips Technologies) and analysed off-line utilising QLAB software.

Results:

The indication for 3DTEE

\begin{tabular}{l|c}
\hline Indication & Number of procedures \\
\hline Subacute bacterial endocarditis & 12 \\
\hline Young stroke & 11 \\
\hline Prosthetic valve dysfunction & 22 \\
\hline Congenital heart disease & 15 \\
\hline Submitral aneurysms & 6 \\
\hline Aortic root complications & 6 \\
\hline Mitral valve disease & 13 \\
\hline Cardiomyopathy & 2 \\
\hline
\end{tabular}


3DTEE was performed with no complications and good patient tolerability of the probe. 3DTEE proved to be of particular benefit in the following: ( I) Delineation of the size and margins of ASD's resulting in improved assessment of suitability for device closure; (2) Localisation and severity of paravalvular mitral prosthetic ring leaks; (3) Definition of prosthetic leaflet mobility and thrombosis; (4) Anatomical characterisation and spatial resolution of submitral aneurysms; (5) Assessment of aortic root complications; and (6) Determining the mechanisms and suitability for mitral valve repair.

Conclusion: 3DTEE is a valuable imaging modality especially in patients with complex anatomy where it has a superior spatial resolution and in those with prosthetic mechanical valves.

\title{
Echocardiographic follow-up of tricuspid annuloplasty with Edwards $\mathrm{MC}^{3}$ ring

\begin{abstract}
Warner Mampuya, Marie-Claude Brochu, Marianne Coutu, Doan Hoa Do, Btissama Essadiqi, Paul Farand,
\end{abstract} David Greentree, Denise Normandin and Serge Lepage
}

Centre Hospitalier Universitaire De Sherbrooke, Sherbrooke, Québec, Canada

Background: Tricuspid regurgitation (TR) occurs mainly from tricuspid annular dilatation as a result of left or right heart pathologies. If present at the time of left heart surgery, significant TR is usually addressed with ring annuloplasty because TR does not reliably resolve after successful left heart surgery and reoperations for recurrent TR carry high mortality rates. Edwards $\mathrm{MC}^{3}$ ring have become the predominant device used in tricuspid valve annuloplasty (TVA).

Methods and results: We analysed short- and mid-term clinical and echocardiographic parameters of 33 consecutive patients (mean age $7 \mathrm{7I} \pm$ 8years) who underwent TVA with Edwards MC $^{3}$ system from February 2006 to April 2009. Two sizes of MC $^{3}$ rings were used: $28 \mathrm{~mm}(\mathrm{n}=9)$ and $26 \mathrm{~mm}(\mathrm{n}=24)$. Short-term echocardiographic studies at $3.1 \pm 1.9$ months after surgery showed a reduction of TR grade from $2.8 \pm 0.7$ to $1.4 \pm 0.8(p<0.00 \mathrm{I})$ and systolic pulmonary artery pressure $(\mathrm{sPAP})$ from $63 \pm 2 \mathrm{mmHg}$ to $42 \pm 13 \mathrm{mmHg}(\mathrm{p}<0.00 \mathrm{I})$ while revealing a peak and mean tricuspid gradients (TG) of $6.8 \pm 2.9 \mathrm{mmHg}$ and $3.0 \pm 1.3 \mathrm{mmHg}$. Compared to short-term evaluation, mid-term echocardiographic studies at $27.3 \pm 11.8$ months showed unchanged TR grade and sPAP. There was a decreasing trend of TG with a peak and mean TG of $5.3 \pm 2.7 \mathrm{mmHg}$ and $2.3 \pm 1.3 \mathrm{mmHg}$, respectively $(p=0.013$ and $p=0.099)$. The mean TG was significantly related to TR grade $(r=0.62, p=0.004)$, sPAP $(r=0.46, p=0.045)$ and age $(r=-0.50 p=0.026)$. There was no correlation between the mean $T G$ and the ring size $(r=-0.277, p=0.248)$.

Conclusion: The Edwards $M C^{3}$ annuloplasty system is very effective in correcting TR. The application of $M C^{3}$ rings creates significant tricuspid gradient which haemodynamic significance is not yet understood.

\section{A large retrospective cohort study of aortic stenosis progression}

\author{
Warner Mampuya, Marie Claude Brochu, Doan Hoa Do, Btissama Essadiqi, Paul Farand and Serge Lepage
}

Centre Hospitalier Universitaire De Sherbrooke, Sherbrooke, Québec, Canada

Background: Calcific aortic stenosis (AS) is the most common valvular lesion in the elderly. Its progression is quite variable and hard to predict in an individual patient. Many studies have identified some risk factors for AS progression. The aim of this retrospective study was to analyse the impact of those risk factors in a large cohort.

Methods and Results: We identified I 259 patients (mean age $70 \pm 14$ years) who had echocardiographic follow up for "pure" AS between 1995 and 2009. Various clinical, echocardiographic, and biochemical variables were related to the change in aortic valve area (AVA). The mean follow-up time was $47 \pm 31$ months. The annual rate of reduction in AVA was $0.07 \mathrm{~cm}^{2}$ or $4 \%$ per year. The reduction in AVA per year was significantly related to age $(r=0.1 \mathrm{I}, \mathrm{p}<0.00 \mathrm{I})$, initial AVA $(r=0.30, P<0.00 \mathrm{I})$, the annual increase of mean aortic valve gradient (AVG) $(r=0.32, P<0.00 \mathrm{I})$ and left ventricular (LV) outflow tract diameter $(r=0.10, P=0.00 \mathrm{I})$. There was no significant correlation with biochemical markers including serum calcium, phosphate, creatinine and lipids. Patients with a rate of reduction in AVA faster than the mean were older 
(72 \pm 12 years vs. $67 \pm 14$ years, $p<0.00 \mathrm{I})$ and had more coronary artery disease (72\% vs. 65\%, $p=0.009)$. These rapid progressors had greater initial AVA $\left(1.62 \pm 0.55 \mathrm{~cm}^{2}\right.$ vs. $\left.1.48 \pm 0.45 \mathrm{~cm}^{2}, p<0.00 \mathrm{I}\right)$, greater LVOT $(2.17 \pm 0.22 \mathrm{~cm}$ vs. $2.13 \pm 0.25 \mathrm{~cm}, p=0.04)$, higher peak AVG $(34 \pm 19 \mathrm{mmHg}$ vs. $31 \pm 17 \mathrm{mmHg}, \mathrm{p}=0.012)$ and mean AVG $(19 \pm 12 \mathrm{mmHg}$ vs. $17 \pm 10 \mathrm{mmHg}, \mathrm{p}=0.014)$. Stepwise Logistic regression identified initial AVA and age as independent predictors of amount of AVA reduction per year.

There were 193 combined events of all causes mortality $(n=31)$ and aortic valve replacement $(A V R, n=162)$. While there was no significant difference in the rate of all cause mortality between the rapid and slow progressors (2.4\% vs. 2.5\%, p=0.89), the rate of AVR was significantly higher in the former group ( $18.4 \%$ vs. $7.4 \%, p<0.00 \mathrm{I}$ ). These combined events happened earlier in the rapid progressors group ( $4 \mathrm{I} \pm 26 \mathrm{months}$ vs. $53 \pm 34$ months, $p<0.00$ I).

Conclusions: Age and initial AVA seem to be primary determinants of AS progression. Patients with rapid progression of AS are older and present with larger initial AVA. They have a higher AVR rate and are operated earlier. These findings can be helpful in the formulation of strategies for follow-up and management of AS patients.

\section{A case of ventricular non-compaction with sub-mitral aneurysms}

\section{M.R. Matshela}

Department of Cardiology, Inkosi Albert Luthuli Central Hospital, Durban, South Africa

Non-compacted ventricle is a rare form of cardiomyopathy caused by embryonic arrest of normal endocardial and myocardial embryogenesis. It is often associated with congenital heart defects, however, there no clear association between non-compacted LV with sub-mitral aneurysms.

This is to show a 29-year old African male who was initially assessed at our cardiac clinic a year ago complaining of progressively worsening dyspnoea for one year, fast and irregular palpitations, atypical chest pains and body swelling.

Physical examination revealed signs of myopathic ventricle with biventricular failure, severe mitral and tricuspid regurgitation and pulmonary hypertension.

His initial ECG revealed sinus tachycardia with a normal axis. Baseline bloods were unremarkable, however, CMV/EBV/Parvo were positive. The CXR revealed increased cardiothoracic ration with pulmonary congestions with features of pulmonary hypertension. Transthoracic echocardiogram revealed dilated and non-compacted RV and LV with moderately impaired LV contractility with an EF of 44\%. Severe mitral and tricuspid regurgitations, PAS of $56+10 \mathrm{mmHg}$. A large aneurysm was noted along the body of posterior mitral leaflet. Transoesophageal echocardiogram revealed a large submitral aneurysm extending to the posterior mitral leaflet with flow noted within it with severe mitral and tricuspid regurgitation and non-compacted ventricle.

CTchest revealed features of lobulated submitral aneurysms with biatrial and biventricular dilatation.

The patient was subjected to sub-mitral aneurysm repair and mitral valve replacement with 30/33 OnX prosthesis and made an uneventful post-operative recovery.

It might have been an incidental finding of submitral aneurysms with non-compacted ventricle, however, other possible causes of submitral aneurysms were excluded including infective endocarditis.

The surgical approach to sub-mitral aneurysms repair is quite controversial but has shown to good long-term outcome. 


\section{A collection of cases presenting with carcinoid syndrome and carcinoid heart disease}

\section{M.R. Matshela}

Department of Cardiology, Inkosi Albert Luthuli Central Hospital, Durban, South Africa

Carcinoid tumours were first described more than 100 years ago at autopsy. Heart disease is a rare form of valvular heart disease and cardiac manifestations is found on echocardiography in more than $50 \%$ of these patients.

I am showing two cases of carcinoid heart disease which we had in our centre. The first is a 78-year old African male who presented with constitutional symptoms, also symptoms and signs of right heart failure. He had features of severe tricuspid regurgitation on examination.

The suspicion of carcinoid heart was only entertained during echocardiography which revealed thickened tricuspid leaflets, marked failure of coaptation with a torrential tricuspid regurgitation and dilated heart. His 5HIAA level was more than 10 times the upper limit of normal. IM octreotite revealed a focus of increased activity in the pelvis and left lobe of the liver. Despite this the patient declined further management and demised a year later.

The second patient is a 32-year old Indian male presented with carcinoid symptoms and features of right sided heart failure, severe tricuspid regurgitation and moderate pulmonary regurgitation. 5HIAA was markedly elevated, positive octreotite scan and CT abdomen revealed multiple large mets in his liver. Echocardiographic features were classical both tricuspid and pulmonary valves involvement. His symptoms improved markedly on octreotite and antifailure medications. He awaits valve surgery.

Outcomes in carcinoid patients with or without heart disease showed shortened life expectancy in patients with cardiac involvement.

Patients with advanced right heart failure are a particularly high risk group, so early detection has a very important prognostic implications in these patients.

\section{Protein Kinase $\mathrm{C}$ epsilon protection against hypoxia requires HIF I alpha and inhibiton of GSK3 beta}

\section{Joy McCarthy, Amanda Lochner, Michael Sack, M. Faadiel Essop and Lionel H. Opie}

Hatter Cardiovascular Research Institute, Faculty of Health Sciences, University of Cape Town, South Africa

PKC $\varepsilon$ plays a central role in cardioprotection. Sub-proteome analysis demonstrated co-localisation of activated cardiac PKC $\varepsilon$ with metabolic, mitochondrial and stress proteins including hypoxia inducible factor I alpha (HIF-Ia) and heat shock proteins. PKC $\varepsilon$ is activated by a number of kinases including Akt. Although PKC $\varepsilon$ inactivates GSK3ß by phosphorylation thereby modulating glycogen metabolism, hypertrophy and HIF-I a, there is no established mechanistic link between PKC $\varepsilon$, p-GSK3ß and HIFI-a.

We hypothesize that constitutive activation of cardiac restricted PKC $\varepsilon$ (aPKC $\varepsilon$ ), and its downstream targets GSK3B/HIF- Ia, induces adaptive cardiac mitochondrial remodeling in response to 14 days hypobaric hypoxia.

Methods and results: aPKC $\varepsilon$ mice and wildtype (WT) controls were exposed to 14 days of chronic hypobaric hypoxia (I I\% O2). Mitochondrial function (mitochondrial respiration, membrane potential, ADP:O ratio, proton leak) and ultrastructure (electron microscopy), ATP synthesis and cardiac function were measured versus controls. We also evaluated myocardial peptide levels of p-GSK3ß and HIF-Ia. Mitochondrial ADP-dependent respiration, ATP production and mitochondrial membrane potential were reduced in chronically hypoxic wildtype cardiac mitochondria but maintained in hypoxic aPKC $\varepsilon$ transgenic mitochondria. Electron microscopy revealed a hypoxia-associated increase in mitochondrial number in controls versus unaltered mitochondrial content in aPKC $\varepsilon$ hearts. Concordantly, exposure to hypoxia diminished left ventricular work in wildtype mice but not in aPKC $\varepsilon$ mice. SDS-PAGE analysis confirmed elevation of p-GSK3B, HIFI-a and some downstream PKC $\varepsilon$ targets in hypoxic hearts versus WT.

Conclusion: We conclude that PKC $\varepsilon$-mediated cardiac adaptation to chronic hypobaric-hypoxia embraces maintenance of mitochondrial homeostasis, integrity and function. We propose that p-GSK3B/HIF-I a activates this paradigm. 


\section{Clinical outcomes of patients undergoing major cardiothoracic surgery infected with the Human Immunodeficiency Virus (HIV)}

\section{S. Naidoo and A. Reddi}

Department of Cardiothoracic Surgery, University of KwaZulu-Natal, Durban, South Africa

Background: The results of major cardiothoracic surgery in patients infected with HIV remains largely unknown. Antiretroviral therapy has lead to a more chronic evolution of HIV with improved life expectancy and quality of life. We report our experience with these patients with an emphasis on selection and operative outcomes.

Methods: A prospective study of I 50 patients from August 2006 to December 2009 was conducted. A database including albumin, BMI, CD4 counts, viral load, Risk Scoring Systems and complications was maintained. Patients for elective surgery were placed on 3 months of HAART if CD4 counts were below 200.

Results: Operations done were 48 pneumonectomies; 46 lobectomies; 30 mitral valve replacements; 20 double valve replacements; 5 aortic valve replacements and I OPCABG. Complications included 3 hospital acquired pneumonias, I CVA and I bronchopleural fistula.

Conclusions: No operative mortality and excellent early outcomes. General health status and pre-operative risk most closely correlated with complication rates. Elective surgery can be safely carried out on patients with HIV and those with CD4 counts below 200 provided that they are on HAART.

\section{An unusual complication of aortic valve prolapse: Adherence of aortic cusp to the tricuspid valve apparatus}

\section{K. Naidoo and K.M. Vanderdonck}

Charlotte Maxeke Johannesburg Academic Hospital and University of the Witwatersrand, South Africa

The vast majority of cases of a ventricular septal defect (VSD) and mild aortic incompetence may be successfully managed by addressing the VSD alone. This often attenuates the progression of the aortic incompetence. We present the case of such a two year old patient with a most unusual anomaly mandating aortic valve repair following VSD closure, despite only mild aortic incompetence preoperatively.

\section{Malignant degeneration of intrapulmonary juvenile laryngeal papillomatosis with intracardiac extension: A case report}

\section{K. Naidoo and K.M. Vanderdonck}

Charlotte Maxeke Johannesburg Academic Hospital and University of the Witwatersrand, South Africa

Primary and secondary intracardiac neoplasms are rare entities. We present the first reported case of an eight year old patient with disseminated congenital trachea-bronchial papillomatosis acutely presenting with a threatened limb and an intracardiac mass. Despite antibiotic therapy and anticoagulation, this mass increased in size and re-embolised. Surgical debulking was therefore undertaken at which time the mass was noted to originate from a pulmonary vein. Histopathological examination revealed a squamous cell carcinoma with superadded infected thrombus. 


\section{Pulmonary arteriovenous malformations: The forgotten right-to-left shunt}

\section{K. Naidoo}

Charlotte Maxeke Johannesburg Academic Hospital and University of the Witwatersrand, South Africa

The differential diagnosis of infantile central cyanosis is limited. We present the case of a twenty month old patient who in the presence of a structurally normal heart, was extensively investigated over a protracted period of time, misdiagnosed and incorrectly treated. The diagnosis of diffuse, unilateral pulmonary arteriovenous malformations causing congestive cardiac failure was eventually made.Embolotherapy was attempted but ultimately failed due to the advanced stage of the disease. Surgical resection with conservative margins was successfully performed thereafter with an excellent clinical outcome.

\section{Spectrum of heart disease in Abuja, Nigeria}

\section{Dike B. Ojji, Ajayi Samuel, Alfa Jacob, Mamven Manmark and Alabi Peter}

Cardiology Unit, Department of Medicine, University of Abuja Teaching Hospital, Gwagwalada, Abuja, Nigeria

Introduction: Inspite of the fact that heart disease is gradually becoming a major cause of morbidity and mortality in sub-Saharan Africa, there is a dearth of data on the spectrum of heart disease in this part of the world. We therefore decided to study the spectrum of heart disease in an urban set-up in Nigeria.

Methods: Every consecutive patient presenting to the cardiology clinic of University of Abuja Clinic from April to 2006 and April 2010 were recruited. Their demographic, clinical and laboratory data were obtained.

Results: I 526 subjects were studied. Primary hypertension and hypertensive heart disease were the commonest diagnosis made in $64.4 \%$ of the cases. $73.2 \%$ of these hypertensive heart disease subjects were either overweight or obese, $10.7 \%$ were diabetic, $5.8 \%$ had suffered a cerebrovascular accident, $4.4 \%$ had one form of arrhythmia or the other and $1.6 \%$ had chronic kidney disease. Heart failure accounted for $31.1 \%$ of the total subjects studied, and hypertension was responsible for heart failure in $60.6 \%$ of cases while coronary artery disease was the cause of heart failure in only $0.42 \%$ of cases. Adult congenital heart disease was present in $0.46 \%$ of the total subjects studied.

Conclusion: Primary hypertension is the commonest risk factor for heart disease in this part of the world. Most cases of heart failure are non-ischaemic with hypertension being the leading cause. Coronary artery disease still remains an uncommon cause of heart failure in Abuja, Nigeria. 


\section{Two transplanted hearts - with one common arrhythmia: How permanent are scars and lines of block in the heart?}

\section{Andrzej Okreglicki}

Cardiac Clinic, University of Cape Town, Groote Schuur Hospital, Cape Town, South Africa

Introduction: Long-term benefit of ablation of arrhythmias such as atrial fibrillation (AF) and flutter is dependent on reliability and permanence of lines of conduction block. However, conduction across surgical scars, which are usually considered to be the most complete and reliable conduction barrier, may occur e.g. in heart transplantation between recipient and donor.

Methods: This case reports the phenomenon of cross-scar signal conduction uniquely in a "piggy-back" double heart transplant patient occurring late after surgery resulting in one common arrhythmia simultaneously in both transplanted hearts.

Results: A 44-year man followed up at our transplant clinic for 30 years presented with palpitations. He had a "piggy-back" heterotopically placed heart transplant in 1980, explantation of his own heart and replacement with an orthotopically placed heart transplant in 1984. The ECG showed an atrial tachycardia with variable AV conduction, at times I:I and heart rates of 200. LV function was normal and there was no evidence of acute rejection. At EP study with Ensite mapping, most of the RA was made up of the functional donor orthotopic atrium and an "island" of atrial tissue of the heterotopic heart with a tachycardia at 260bpm and intermittent conduction between them. A macroreentrant tachycardia was mapped and successfully ablated with no recurrence.

Conclusions: This illustrates that scars are not permanent lines of conduction block. This poses concern regarding ablation in AF and claims of cure particularly if the potential of recovery of conduction exists.

\section{Atrioventricular Nodal Reentry Tachycardia (AVNRT): Age and sex determined variations in AV node electrophysiology}

\section{Andrzej Okreglicki and Carl Russouw}

Cardiac Clinic, University of Cape Town, Groote Schuur Hospital, Cape Town, South Africa

Introduction: Age and gender may influence the function of the circuit of pathways in AVNRT and account for differences in demographic presentation.

Methods: All electrophysiological studies with complete records of patients who underwent AVNRT ablation in our institution's EP laboratory 2001 to 2008 were reviewed looking at gender differences in clinical and electrophysiological characteristics. Females were further analysed according to age: child bearing ( $15-50$ years) and older (>50 years).

Results: Complete records of 78 patients were available: 58 (74.4\%) females and 20 (25.6\%) males with ages $12-78$ yrs. At EP study, no gender-based differences in refractory period (RP) were noted. However, on analysis of the female group by age, the slow pathway functional RP was shown to be significantly longer in the younger females: $519 \pm 141 \mathrm{~ms}$ vs $426 \pm 73 \mathrm{~ms}(\mathrm{p}<0.02)$.

Conclusions: Apart from the commonly recognised higher incidence of AVNRT amongst women, no significant clinical difference was noted between males and females in this retrospective audit except for the group of younger "child-bearing" females having significant electrophysiological differences in the slow AVN pathway when compared with the older group. This finding may explain their increased incidence of AVNRT compared to males which does not occur amongst the older men and women. This suggests sex hormones may indeed play a role in AVNRT and that once women become post-menopausal, the electrophysiological properties of the AV node start to resemble that of males accounting for the near equivalent incidence of AVNRT in females $>50$ years and men. 


\section{Ebstein's anomaly and Down syndrome: A case report}

\section{Lungile Pepeta and Samina Mahmud Yakoob}

Division of Paediatric Cardiology, Dora Nginza Hospital, Port Elizabeth Hospital Complex and Walter Sisulu University, Port Elizabeth, South Africa

Ebstein's anomaly was first reported by Wilhelm Ebstein in 1866. This cardiac anomaly is characterized by significant (more than $8 \mathrm{~mm} / \mathrm{m}^{2}$ body surface area) apical displacement of the tricuspid valve. It is very rare, as it occurs in 1 in 200000 patients. On the other hand, Down syndrome is the commonest chromosomal syndrome, and it occurs in I in 800 to 1 in 1000 live births. The incidence increases with advanced maternal age. Congenital cardiac lesions are very common in patients with Down syndrome as they are seen in 40-50\% of patients with this syndrome, with the commonest being atrio-ventricular septal defect. A combination of Ebstein's anomaly and Down syndrome is extremely rare, with only five case reports, to the best of our knowledge. A case report and brief review of the other cases will be presented.

\section{Utility of 3D Echocardiography for the diagnosis of Congenital Submitral Aneurysms}

\section{F. Peters, A. Gikonyo and M.R. Essop}

Division of Cardiology, Chris Hani Baragwanath Hospital, University of the Witwatersrand, Johannesburg, South Africa

Background: Congenital submitral aneurysms (SMA) are not an uncommon cause of mitral regurgitation in sub-Saharan Africa. They usually arise from the mitral annulus extending in any direction to compress a variety of cardiac structures. Precise anatomical definition is important for the successful surgical management of SMA.

Aim: To evaluate the utility of transthoracic 3D echocardiography for the assessment of SMA preoperatively.

Methods: Between September 2009 and April 2010, 8 consecutive patients with suspected SMA underwent routine transthoracic 2D and 3D echocardiography using a Philips IE 33 system. Three scanning modalities, full volume, 3D zoom and multiplane reconstruction (MPR) were utilised to define the origin and size of the aneurysm and presence of associated complications.

Results: All patients had single aneurysms. The origin of the SMA in relation to the mitral annulus was anterior in 3, posterior in 4 and at the medial commissure extending in both directions in I patient. The neck as well as the spatial extent of the aneurysm was identified in all patients. Clot lined the aneurysm in 2 patients, rupture into the left atrium occurred in 3 patients and one patient had an associated congenital sinus of Valsalva aneurysm. Visualisation from the left ventricular perspective was superior to analysis from the left atrial perspective except in cases of rupture. 3D echocardiography resulted in reclassification compared to 2D in 3 patients.

Conclusion: Transthoracic 3D echocardiography is an effective non-invasive modality to accurately assess SMA and its spatial relationships and has important implications when considering surgical correction. 


\section{Isolated left ventricular non compaction: A single centre experience}

\section{Ferande Peters, Claudia Dos Santos, Krinesh Naidoo, Chris Zambakides and Rafique Essop}

Division of Cardiology, Chris Hani Baragwanath Hospital, University of the Witwatersrand, Johannesburg, South Africa

Background: Isolated left ventricular non-compaction (ILVNC) is a rare congenital cardiomyopathy caused by intrauterine arrest of normal compaction of the myocardium. Major complications include heart failure, ventricular arrhythmias and cardio-embolism.

Aim: To determine the clinical characteristics and echocardiographic features of ILVNC.

Methods: A prospective study of 29 consecutive patients who met the Jenni criteria for ILVNC. Detailed echocardiography was performed in all patients and analysed using current ASE recommendations.

Results: The mean age was 47 years, 61 \% were male and 60\% of patients were NYHA class 2/3 on conventional medical therapy. One patient had an ICD implanted following a ventricular storm. Thrombus in the left ventricle (LV) was found in one patient with one other having had a past cardio-embolic event. Five patients were on Warfarin. Involvement of the apex, apico-inferior and apico-lateral walls of the LV occurred in all patients. Mean LV end-diastolic diameter was $62 \mathrm{~mm}$ and ejection fraction $25 \%$. Two patients had a normal LVEF. Moderate or severe mitral regurgitation was found in 62\%. Right ventricular (RV) abnormalities were common with RV dilatation in 65\%, significant tricuspid regurgitation in $61 \%$ and pulmonary hypertension in $61 \%$. The tricuspid S-wave could be adequately analysed in 21 patients and averaged $8.6 \mathrm{~cm} / \mathrm{s}($ Normal $>12 \mathrm{~cm} / \mathrm{s})$

Conclusion: ILVNC is characterised by significant abnormality of both left and right heart function. Long term follow-up is required to accurately determine clinical outcomes.

\section{Gender specific differences in metabolic gene expression and left ventricular function in isoproterenol induced Left Ventricular Hypertrophy (LVH)}

\section{Vernice R. Peterson, Tlangelani Maswanganyi and Siyanda Makaula}

Cardiovascular Pathophysiology and Genomics Research Unit, School of Physiology, Faculty of Health Sciences, University of the Witwatersrand, Johannesburg, South Africa

Background: Male and female rat hearts exhibit dissimilar degrees of susceptibility to cardiovascular diseases. Also, metabolic remodeling is associated with LVH. However, it remains unclear whether gender specific differences in metabolic response can be attributed to differential responses in LVH and cardiac dysfunction. The aim of this study was to determine whether isoproterenol induced LVH would exhibit a gender specific pattern in metabolic gene expressions and cardiac dysfunction.

Methods: Male and female Sprague-Dawley rats were assigned to control group and isoproterenol group (ISO-M and ISO-F). The control groups received saline injections and isoproterenol groups received subcutaneous injections of $0.02 \mathrm{mg} \mathrm{kg}^{-1}$ day- $^{-1}$ of isoproterenol for five weeks. Echocardiography was performed to assess cardiac function. Total heart mass (HM) and left and right ventricular masses (LVM and RVM) were weighed to determine LVH. Quantitative real-time PCR analysis was performed to assess metabolic gene expressions of AMPKa $a_{2}$, PPARa, Tfam and NRF-I.

Results: HM and LVM were increased in both ISO-M and ISO-F groups compared to their respective controls ( $P<0.05$ for both). However, ISO-F also showed an increase in RVM ( $p<0.05)$. ISO-M showed a reduction in endocardial fractional shortening (FSend) compared to controls $(\mathrm{p}<0.0 \mathrm{I})$ however, FSend was unchanged in the ISO-F group. In ISO-M, AMPKa ${ }_{2}$, PPARa and Tfam gene expressions were significantly reduced compared to their controls $(p<0.05$ for all). In contrast, ISO-F showed an increase in the expression of NRF-I $(p<0.0 \mathrm{I})$.

Conclusions: Susceptibility to isoproterenol induced cardiac dysfunction is associated with reduction in the expression of key metabolic regulatory genes in males; however, these changes were not observed in females. The reduced cardiac susceptibility to isoproterenol in females could be explained by NRF-I. 


\title{
Inflammatory marker comparison between patients with acute coronary syndrome under-going on-pump versus off-pump coronary artery bypass graft surgery
}

\author{
H. Potgieter ${ }^{*}$ F.E. Smit ${ }^{\#}$ L. Botes ${ }^{\dagger}$, W.M.L. Neethling ${ }^{* *}$ \\ "National Health Laboratory Services, Universitas Hospital, Bloemfontein, South Africa \\ \#Department of Cardiothoracic Surgery, University of the Free State, Bloemfontein, South Africa \\ tSchool of Health Technology, Central University of Technology, Bloemfontein, South Africa \\ **Department of Cardiothoracic Surgery, Fremantle Heart Institute, Perth, Western Australia
}

\begin{abstract}
Introduction: Previous studies have compared outcomes in on-pump and off-pump CABG (Coronary Artery Bypass Graft) surgery patients. Both on-pump and off-pump CABG surgical procedures elicit systemic inflammatory responses, sometimes leading to SIRS (Systemic Inflammatory Response Syndrome). This study compared changes in peri-operative inflammatory markers and clinical outcomes in on- and off-pump (CABG) surgery in patients presenting with Acute Coronary Syndrome (ACS).
\end{abstract}

Methods: A prospective analytical observational study was conducted. Sixty patients presenting with ACS undergoing CABG surgery were recruited ( $n=30$ on-pump and $n=30$ off-pump). Inflammatory markers (full blood count (FBC), procalcitonin (PCT), C-reactive protein (CRP), Interleukin-6 (IL-6) and tumor necrosis factor alpha (TNF-a)) for both groups were analysed and compared pre-operatively and post-operatively. Clinical outcomes were compared between on-pump and off-pump surgery groups.

Results: When compared to the off-pump group the on-pump CABG group showed a significant increase in acute phase cellular responses in: White Cell Count (WCC) at 24h $(p=0.0076 \mathrm{I}), 48 \mathrm{~h}(\mathrm{p}=0.01520)$ and $72 \mathrm{~h}(\mathrm{p}=0.00004)$, neutrophils at 24h $(p=0.17422), 96 \mathrm{~h}(\mathrm{p}=0.186 \mathrm{II})$ and $120 \mathrm{~h}(p=0.12872)$, lymphocytes at $48 \mathrm{~h}(p=0.04829)$ and at $96 \mathrm{~h}(p=0.01982)$ and PCT at $24 \mathrm{~h}(p=0.008 \mathrm{II}), 48 \mathrm{~h}(p=0.00966)$ and $72 \mathrm{~h}(p=0.01823$. However, the IL-6 levels found in the off-pump CABG group were significantly higher at $96 \mathrm{~h}(p=0.05352)$ and $120 \mathrm{~h}$ $(p=0.09729)$.

Conclusion: No difference in clinical outcomes could be demonstrated between the on- and off-pump CABG surgery patients despite a more pronounced cellular response in the on-pump group. The IL6 response in the off-pump group has previously been noted, but mechanisms remains unclear.

\section{Cardiovascular risk profile of young Indians}

\section{Rosaley Prakaschandra and Datshanna Naidoo}

Department of Cardiology, Inkosi Albert Luthuli Central Hospital, Durban, KwaZulu-Natal, South Africa

Premature CHD has been reported to be increasingly common in the Indian community, and this has been linked to genetic predisposition to disease. However much of the familial predisposition could be related to lifestyle patterns from an early age.

Objectives: The aim of this study was to document the prevalence of risk factors in younger subjects <34 years in this community.

Method: A randomised sample was selected for study and stratified into gender and age groups in the Phoenix community and the major risk factors measured. The presence of the metabolic syndrome (MS) was determined using NCEP ATP III and IDF criteria.

Results: Of the 297 participants studied, impaired fasting glucose was present in 3\% of the 15-24 age group, and rose to 5\% in the 25-34 age group. In the same groups impaired GTT was present in 5\% and 16 respectively. Elevated total cholesterol (>5. I $8 \mathrm{mmol} / \mathrm{L})$ was observed in 15\% of the 15-24 year olds, and $41 \%$ of the $25-34$ age group, with the latter group also comprising $20 \%$ with abnormal HDL (<I.0mmol/L). There was a 7\% prevalence of MS in the 15-24 age group and this increased to 23\% (ATPIII) - 29\% (IDF) in the 25-24 year olds. The BMI was $>25$ in all subjects in the 15-24 and in $94 \%$ of the $25-34$ year groups with MS.

Conclusion: There is high prevalence of impaired glucose metabolism in young subjects in this community, not reflected by the fasting blood glucose. There is also a very high prevalence of MS in this randomised sample. Risk factors emerge as early as 15 years, with a sharp increase in the 25-34 year age group, largely driven by obesity. 


\section{Surgical esoteria}

\section{Reddy, F.C. Cardio and A. Reddi}

Department of Cardiothoracic Surgery, Nelson R Mandela School of Medicine, University of KwaZulu-Natal and Inkosi Albert Luthuli Central Hospital, Durban, South Africa

In addition to the conventional pathology referred to a cardiothoracic surgical unit, on occasion, esoteric surgical disease is encountered; often with dramatic clinical presentations, unusual radiographic manifestations and noteworthy intra-operative findings.

Using a series of case vignettes, the clinical, radiographic and intra-operative features of the following pathologies will be discussed:

- An aneurysm of the sinus of Valsalva with massive interventricular septal penetration presenting with complete heart block

- An aneurysm of the proximal descending thoracic aorta with complete erosion of the left main bronchus presenting with haemoptysis

- A metastatic cardio-pulmonary osteosarcoma mimicking a left atrial myxoma ten years after ablation of the lower limb primary tumour

- Idiopathic pulmonary vein thrombosis presenting with massive haemoptysis ${ }^{(1)}$

Following each clinical scenario, a brief review of the current surgical literature and the principles of surgical therapy will be highlighted.

References: I. Alexander G, Reddi A, Reddy D. Idiopathic pulmonary vein thrombosis: A rare cause of massive haemoptysis. Ann Thorac Surg 2009;88:28I-3.

\section{A case control study of cardiovascular health in chemical war-disabled Iranian victims}

\section{Atooshe Rohani and Vahid Akbari}

Department of Medicine, Division of Cardiovascular Medicine, Yasooj University of Medical Science, Iran

Background: Sulfur mustard (SM) is an alkylating chemical warfare agent that was widely used during World War I and in Iran-Iraq war between 1983 and 1988. Sulfur mustard exposure leads to various late complications. The aim of this study was to determine late cardiovascular effects of sulfur mustard in war-disabled Iranian victims.

Methods: This was a retrospective cohort case control study of 50 patients with symptoms of sulfur mustard exposure (during 1983-1988) who are living now in Yasooj city (2006-2008) and 50 who were attending in Iran-Iraq war and did not have chemical injury. We performed exercise stress test and echocardiography on all of them. The chi-square test was used for data analysis.

Results: The study group comprised of 100 males within the age $45.6 \pm 6.2$ years. 50 of them were exposed to high dose SM at least 24 years ago. Their mean weight was $67.2 \pm 8.3 \mathrm{~kg}$. In chemical war injury group two patients (4\%) has positive exercise stress test and in coronary angiography both of them had 2-vessel disease. They underwent successful angioplasty. LV diastolic abnormality (relaxation impairment) was detected in $23 \%$ of subjects. There were no considerable conductive abnormalities. One of them had severe mitral regurgitation that had normal coronary angiography and referred for mitral valve replacement. In another group, there were no considerable valvular or conductive abnormalities. About 5\% of them had LV diastolic abnormality (relaxation impairment) $(p=0.02)$. All of them had normal exercise stress tests. Conclusions: Cardiovascular disability is another late complication in chemical war-disabled Iranian victims. Diastolic dysfunction was the most common abnormality in both groups. 


\title{
The prevalence of HIV amongst care-givers of children undergoing congenital heart surgery
}

\section{H. Sathekge, K. Naidoo and K.M. Vanderdonck}

Charlotte Maxeke Johannesburg Academic Hospital and University of the Witwatersrand, South Africa

Patients underdoing cardiac surgery have not been immune to the epidemic of HIV/AIDS in South Africa. Many patients undergoing congenital and paediatric cardiac surgery, are afflicted in various ways by HIVIAIDS. A retrospective review of the HIV status of care-givers of infants undergoing surgery was performed, representing a randomly selected portion of the population. Approximately $25 \%$ of care-givers of infants undergoing surgery tested positive for HIV. Some theories are submitted.

\section{A not so rare form of heart failure in urban Black Africans: Right heart failure in the Heart of Soweto Study cohort}

\author{
Karen Sliwa*,\#, Melinda Carrington $\#, t$, Sandra Pretorius ${ }^{\#}$ and Simon Stewart ${ }^{\#, t}$ \\ *Hatter Cardiovascular Research Institute, Faculty of Health Sciences, University of Cape Town, South Africa \\ \#Soweto Cardiovascular Research Unit, University of the Witwatersrand, Johannesburg, South Africa \\ †Baker IDI Heart and Diabetes Institute, Melbourne, Australia
}

Background: Preliminary data suggest that right ventricular dysfunction is far more prevalent in urban Black African patients presenting with the syndrome heart failure (HF) than reports from high income countries would suggest.

Methods: Chris Hani Baragwanath Hospital services the Black African community of I.I million people in Soweto, South Africa. A prospective, clinical registry captured data from all de novo cases of structural and functional heart disease presenting to the cardiology unit during 2006 - 2008. We examined the prevalence and characteristics of right heart failure (RHF) in 5328 cardiac cases captured by the registry.

Findings: Overall, there were 2505 cases of HF (47\%); of which 697 (28\%) involved a component of RHF. Despite more women ( $\mathrm{n}=379$ ) overall, men $(n=318)$ were likely to present with RHF (I2\% vs. I5\% of cases; or 1.27, 95\% Cl I.08 - I.49: $p=0.003)$. Black Africans ( $n=642$, 92\%) made up the majority of cases. According to baseline profiling, presentation with RHF was independently associated with being Black

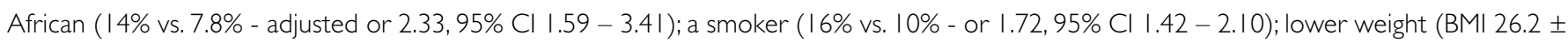
6.2 vs. $28.4 \pm 7.4 \mathrm{~kg} / \mathrm{m} 2$ - or $0.96,95 \% \mathrm{Cl} 0.94-0.97$ ); and less family history of heart disease (I $\%$ vs. | $4 \%$ - or $0.79,95 \% \mathrm{Cl} 0.64-0.96$ ) RHF was the primary diagnosis in $50 \%$ of cases and a common complication to idiopathic and HIV-related dilated cardiomyopathy (I0I cases), 15\%); rheumatic heart disease (99 cases, 14\%); and hypertensive HF (65 cases, 9.3\%). A majority of cases presented with dyspnoea (84\%) and/or palpitations (60\%). Chronic lung disease (I0I cases, I5\% cases), lung pathology linked to TB and HIVIAIDS (7I cases, I0\%) and 26 cases of pulmonary hypertension secondary to connective tissue disease or thrombo-embolism. Clinically significant tricuspid regurgitation was evident in $54 \%$ of cases, mean right ventricular systolic pressure was $53 \pm 19 \mathrm{mmHg}$ and mean left ventricular ejection fraction was $54 \pm 17 \%$. Conclusion: These unique data confirm that RHF is a prevalent condition among Black African patients with heart disease with multiple causes evident. Specific screening and treatment strategies for RHF are indicated for such high risk patient cohorts. 


\title{
Impact of Human Immunodeficiency Virus (HIV)/AIDS on the manifestation of newly diagnosed heart failure in an urban African community: Insights from the Heart of Soweto Study
}

\author{
Karen Sliwa", Melinda Carrington",t, Anthony Becker", Mpiko Ntseke", Sandra Pretorius" and Simon Stewart ${ }^{\#, t}$ \\ *Hatter Cardiovascular Research Institute, Faculty of Health Sciences, University of Cape Town, South Africa \\ \#Soweto Cardiovascular Research Unit, University of the Witwatersrand, Johannesburg, South Africa \\ ${ }^{\dagger}$ Baker IDI Heart and Diabetes Institute, Melbourne, Australia
}

Background: South Africa has more than 5 million people living with HIVIAIDS. Little is known about the impact of the HIV/AIDS epidemic on the manifestation of newly diagnosed cardiovascular disease (CVD) including heart failure from urban African communities.

Methods: Chris Hani Baragwanath Hospital services the Black African community of I.I million people in Soweto, South Africa. A prospective, clinical registry captured data from all de novo cases of CVD presenting to the cardiology unit during 2006 - 2008 . We describe in detail all cases with newly diagnosed CVD where HIVIAIDS was the probable causative factor (e.g. HIV-related cardiomyopathy [CMO]) or where it was most probably an incidental co-morbidity within a population in whom there is a high prevalence of infection. HIV tests were performed when clinically indicated and consent available.

Findings: During the study period, 506 of 5328 cases with newly diagnosed CVD were determined as HIV positive (9.5\%). Women ( $\mathrm{n}=3 \mathrm{I} 3$ cases, 62\%) and Black Africans (488 cases, 96\%) predominated; women being significantly younger than men $38 \pm 14$ vs. $42 \pm 13$ years $(p=0.002)$. The most common primary diagnoses attributable to HIVIAIDS were HIV-related CMO (I 84 cases, $36 \%)$ and clinically significant pericardial effusion (PE)/pericarditis (65 cases, 12.9\%): representing 3.5\% and 1.2\% of all CVD cases. A further 44 cases were diagnosed with comorbid PE; it being present in 22\% ( 109 cases) of all HIVIAIDS cases. Patients with HIV-related CMO presented in significantly older men $(p=0.007)$ and had a lower eGFR ( $p=0.022)$ compared to the overall HIV positive population. Overall, 54\% of all HIVIAIDS cases were prescribed highly active antiretroviral therapy at the time of presentation; those diagnosed with HIV-DCMO being more likely to be on such treatment versus the rest: $127 / 184$ (69\%) vs. 147/322 (46\%) cases, respectively (or 2.85, 95\% Cl I.81 to 3.88).

Conclusion: HIV-related CMO followed by PE/pericarditis is the most common diagnosis in predominantly urban black African patients being HIV positive and presenting with de novo CVD. Specific, treatment and surveillance strategies for HIVIAIDS-related CVD states need to be developed and applied given the size and scope of the problem. 


\section{Prognostic significance of myocardial delayed enhancement in patients with sarcoidosis}

\section{Jan-Peter Smedema, Gabriel Snoep, Robert-Jan van Geuns, Joris Ector, Hein Heidbuchel, Liselot van Erven, Joop Schreur, Gillian Ainsley and Harry Crijns}

Netcare NI City Hospital, Goodwood, South Africa

Background: In patients with sarcoidosis, sudden death is a leading cause of mortality, which may represent unrecognized cardiac involvement. Delayed-Enhanced Cardiovascular Magnetic Resonance (DE-CMR) can detect minute amounts of myocardial damage, and has been demonstrated to accurately diagnose cardiac sarcoidosis (CS). We sought to determine whether myocardial delayed enhancement (MDE) was related to adverse outcome in patients with sarcoidosis.

Methods and results: II 3 patients (34-76 years, mean 52.8 years, 74 men, 80\% Caucasian) with biopsy proven pulmonary sarcoidosis who had baseline DE-CMR were followed for 3-108 months (mean 58.2 months). 26 patients presented with cardiac symptoms, and 87 were screened for cardiac involvement. All patients with MDE had coronary disease excluded by $X$-ray angiography. Endpoints included hospital admission for heart failure, sustained ventricular tachycardia, appropriate device therapy for VT/VF, pacemaker implantation for advanced atrioventricular conduction block, and sudden death. MDE was present in 31 patients (mean 19\%, range 3-39\% of LV mass), mostly involving basal and lateral LV segments (70\%). The transmural and relative left ventricular extent of MDE correlated with severity of systolic left ventricular dysfunction $(p<0.00 \mathrm{I})$, atrio-ventricular $(p<0.05)$ and intraventricular $(p<0.05)$ conduction disease, as well as non-sustained/ sustained VT $(p<0.05)$. During follow up 20 patients had adverse events. MDE strongly correlated with events during follow up (Kaplan-Meier curves, logrank test, $p=0.00025$ )

Conclusions: In patients with sarcoidosis the amount of MDE strongly correlated with the severity of LV dysfunction, conduction disease and ventricular dysrhythmias. MDE predicted adverse outcome during longterm follow up. Therefore, we state that DE-CMR is an important new tool in the risk-assessment and management of patients with CS. 
Prediction of surgical outcomes in acute coronary syndromes: Framework for a proposed integrated risk model

\section{Francis Edwin Smit, Carla Prins, Izak Jonker and Lezelle Botes}

Department of Cardiothoracic Surgery, University of the Free State, Bloemfontein, South Africa

Introduction: Coronary artery bypass grafting (CABG) has achieved excellent results in the treatment of coronary artery disease. Analyses of risk factors and outcome are complicated by the intricate pathophysiology of acute coronary syndromes (ACS) and intra-operative events. Widely used risk scoring systems like the European System for Cardiac Operative Risk Evaluation (EuroSCORE) does not take intra-operative events into account. Integrating pre-operative risk scores with intra-operative events will theoretically provide more accurate information.

\begin{tabular}{c|c|c}
\hline $\begin{array}{c}\text { Currently utilised risk models } \\
\text { Actual course of treatment } \\
\text { and exposures to patient }\end{array}$ & Diagnosis: & Preoperative risk prediction \\
\hline
\end{tabular}

Methods: Descriptive reviews were performed on: (I) currently utilised models quantifying risk for adverse outcomes during pre-operative assessments and; (2) intra-operative factors potentially influencing the outcomes of the surgical treatment of coronary artery disease. Sixty patients were recruited for a prospective cohort study allowing sample analyses of potentially predictive factors related to outcomes. Pre- and intra-operative parameters were analysed in relation to post-operative outcomes.

\begin{tabular}{|c|c|c|c|c|}
\hline Phase I & Phase 2 & Phase 3 & Phase 4 & Phase 5 \\
\hline $\begin{array}{l}\text { Literature review } \\
\text { of existing models }\end{array}$ & $\begin{array}{l}\text { Review additional } \\
\text { potential risk factor }\end{array}$ & $\begin{array}{l}\text { Design prototype model } \\
\text { combining existing models } \\
\text { and additional risk factors }\end{array}$ & $\begin{array}{c}\text { Perform sample } \\
\text { analyses on } 60 \text { patients }\end{array}$ & $\begin{array}{l}\text { Refine and finalise } \\
\text { the proposed model }\end{array}$ \\
\hline
\end{tabular}

\section{Results:}

\begin{tabular}{|l|c|c|c|c|}
\hline Acute coronary syndromes (ACS) & Pre-operative risk factors & $\begin{array}{c}\text { Surgical treatment: } \\
\text { Intra-operative events }\end{array}$ & Outcomes \\
\hline $\begin{array}{c}\text { Atherosclerosis, inflammation and } \\
\text { endothelial dysfunction }\end{array}$ & $\begin{array}{c}\text { Patient history and } \\
\text { other clinical risk factors }\end{array}$ & CABG: Surgical trauma & In-hospital mortality \\
\hline $\begin{array}{c}\text { Plaque disruption, platelet } \\
\text { aggregation, thrombosis/obstruction }\end{array}$ & $\begin{array}{c}\text { Biochemical markers: Markers of } \\
\text { Cardiovascular (CV) risk, clinical or } \\
\text { subclinical CV disease and co-morbid } \\
\text { diseases, myocardial muscle damage, } \\
\text { pro-inflammatory markers }\end{array}$ & $\begin{array}{c}\text { Technique:With/without the use of } \\
\text { cardio-pulmonary bypass }\end{array}$ & Anesthesia \\
\hline $\begin{array}{c}\text { ACS: ST-segment elevation myocardial } \\
\text { infarction, non-ST segment myocardial } \\
\text { infarction and unstable angina }\end{array}$ & Atheroma load as risk factor & $\begin{array}{c}\text { Haemodynamic and tissue } \\
\text { perfusion measurements }\end{array}$ & $\begin{array}{c}\text { Biochemical outcomes: Markers of } \\
\text { myocardial damage and inflammation }\end{array}$ \\
\hline
\end{tabular}

Conclusion: The development of an integrated risk predictive model includes hitherto underutilised intra-operative and other known perioperative risk factors. This allows more accurate risk stratification. An ICU-admission outcomes prediction model can be developed on this data. Continuous assessment of risk factor impact on outcomes improved clinical decision making. 


\section{The effect of oleanolic acid on nitric oxide production in normal and dysfunctional cardiac endothelial cells}

\section{Hans Strijdom, Amanda Genis, Mashudu Mudau, Pretty Murambiwa and Cephas Musabayane}

Division of Medical Physiology, Department of Biomedical Sciences, Faculty of Health Sciences, University of Stellenbosch, South Africa

Dysfunction of the endothelium, characterised by reduced nitric oxide (NO) production, is an early event in the pathogenesis of atherosclerosis. Mechanisms of endothelial dysfunction (ED) include deficient endothelial NO synthase (eNOS) function, oxidative stress and increased inflammation. Interventions aimed at reversing ED could have major therapeutic value in the prevention of atherosclerosis. Anecdotal evidence suggests that traditional medicinal plants possess cardioprotective properties, such as the water berry tree (Syzigium cordatum). The bioactive compound, oleanolic acid (OA), has recently been isolated from S. cordatum leaf extracts. OA has been shown to enhance endotheliumdependent vasodilatation in mesenteric arteries, suggesting a possible role for $\mathrm{OA}$ in increasing NO-production, a property which makes $\mathrm{OA}$ a candidate ED-reversing molecule. This study aimed to investigate the short-term effects ( $5 \mathrm{~min}$ and $20 \mathrm{~min}$ ) of OA (30uM and 40uM) in a model of cardiac microvascular endothelial cells (CMECs) by measuring necrosis, apoptosis and intracellular NO-production. Subsequently, we established a tumor necrosis factor-alpha (TNF-alpha)-induced model of ED and investigated whether OA would reverse the observed reduction in NO-production. Results showed that $\mathrm{OA}$ had no pro-necrotic and pro-apoptotic effects, but 40 uM incubated for 20 min significantly increased baseline NO-production. Incubation of TNF-alpha $(5 \mathrm{ng} / \mathrm{ml})$ for $24 \mathrm{~h}$ induced ED in the CMECs (reduced NO-production, reduced eNOS activation and increased NADPH-oxidase expression). Co-incubation with OA (40uM) significantly increased NO-production to control levels, thereby reversing the NO-lowering effects of TNF-alpha. This study demonstrates a promising role for OA in the reversal of ED by increasing NO-production in CMECs.

\section{Infective Endocarditis in Africa}

\section{J.A.T. Stroebel", F.E. Smit", C Prins*, S.C. Brown" and J. Cloete*}

*Department of Cardiothoracic Surgery, University of the Free State and Universitas Hospital, Bloemfontein, South Africa \#Department of Paediatric Cardiology, University of the Free State and Universitas Hospital, Bloemfontein, South Africa

Background: Infective endocarditis (IE) is an aggressive disease and is associated with considerable mortality and morbidity. In spite of advances in antibiotic therapy the incidence rates of IE in developed countries is rising. Very little, however, is published about infective endocarditis in developing countries.

Methods: We performed a PubMed search for endocarditis publications from Africa as part of a literature review of IE in developing countries. We then performed a retrospective analysis of IE in our adult patient population for the period $2006-2010$ and a retrospective analysis of our paediatric population from 1999 - 2010.

Results: 42 Articles were identified. 25 discussed primary infective endocarditis, in 15 IE was discussed in cardiovascular disease context and 2 were classified as other. Analysis of the literature review and retrospective analysis revealed that a major predisposing risk factor for IE in Africa is rheumatic heart disease (RHD) (incidence 60 - 100\%). There is a low incidence of drug related IE. Poor dental hygiene does not seem to be a common predisposing factor and HIV infection seems to have a limited impact. Mortality ranged from $25 \%$ to $100 \%$ in Africa, and our unit's surgical mortality was comparable to that of developed countries.

Conclusion: It is imperative that we establish preventative programmes for RHD, establish secondary prevention programmes and implement national protocols for the diagnosis and management of IE. Aggressive early surgery improves outcome and therefore we need to expand cardiothoracic surgical services, not only in South Africa but in Africa. 


\section{Carmen Swanepoel, Amsha Ramburan, Lundi Korkie, Craig Kinnear and Johanna Moolman-Smook}

University of Stellenbosch Medical Research Council (US/MRC), Centre for Molecular and Cellular Biology, Health Sciences,

University of Stellenbosch, South Africa

The Copper-metabolism-MURR I-domain-containing-protein 4 (COMMD4) was found to bind in a phosphorylation dependant manner to the $\mathrm{N}$-terminal of CMyBPC, a protein involved in structural assembly and stability of the thick filament as well as the regulation of cardiac contractility. Given that MYBPC3 mutations cause hypertrophic cardiomyopathy (HCM) and that COMMD proteins have been linked to hypertrophy, this protein of unknown function was therefore prioritised for further investigation by identifying its protein binding partners by $\mathrm{Y} 2 \mathrm{H}$ analysis and confirming these interactions via verification assays. Moreover, the functions of COMMD proteins are still unclear, but has been linked to copper metabolism and the ubiquitin-proteasome pathway (UPS). Interestingly, recent studies have shown that the UPS have been implicated in at least MyBPC-derived HCM, whereas dietary copper-depletion has been shown to cause cardiac hypertrophy.

A COMMD4 Y2H-bait construct was generated and used to screen a cardiac cDNA-library. Putative interactors were identified by direct sequencing and analyzed using bio-informatics tools. Eight plausible positive interactors were verified via in vivo co-immunoprecipitations analysis and 3D live cell fluorescent co-localization. The effect of RNAi-mediated COMMD4 knock-down on cMyBPC turnover was also investigated.

These plausible interactors yielded novel insights into the role of COMMD4, implicating it in protein trafficking and turnover and providing support for the link between CMyBPC-related HCM and protein degradation. Therefore, the results to date may shed light on the functioning of $\mathrm{cMyBPC}$ and also present a greater understanding of its role in the pathogenesis of HCM.

\section{Development of a subaortic aneurysm secondary to disseminated tuberculosis in a child}

\section{Farirai F. Takawira*, Jayneel A. Joshi* and Dirk J. du Plessis\#}

"Department of Paediatric Cardiology, Steve Biko Academic Hospital and University of Pretoria, South Africa

\#Cardiothoracic Surgery, Steve Biko Academic Hospital and University of Pretoria, South Africa

Introduction: Subvalvular aneurysms of the left ventricle are very rare and often of uncertain aetiology. We describe a unique case of a child with a tuberculous subaortic aneurysm observed at different stages of development by serial echocardiography.

Case report: A three-year-old boy presented to our institution with a history of coughing, fever, night sweats and generalised body swelling. On examination he appeared chronically ill and was underweight for his age. He had generalised lymphadenopathy, ascites and hepatosplenomegaly. He was tachypnoeic and had intercostal and subcostal recession. The heart sounds were muffled. There were no murmurs, but he was in congestive cardiac failure. Chest radiography demonstrated cardiomegaly, a widened mediastinum and paratracheal lymphadenopathy. The C-reactive protein, lactate dehydrogenase levels and erythrocyte sedimentation rate were elevated. Echocardiography revealed normal intracardiac anatomy with a large organised and loculated pericardial effusion. A biopsy of a supraclavicular lymph node and gastric aspirates confirmed TB. A repeat echocardiogram after six weeks of TB treatment demonstrated a small pouch of $2 \mathrm{~mm} \times 3 \mathrm{~mm}$ in the subaortic area. This had grown into a large subaortic aneurysm three months later. The boy underwent successful cardiac surgery five months after the initiation of TB therapy.

Discussion: Subvalvular left ventricular aneurysms can either be subaortic or submitral. Most of the cases of subvalvular left ventricular aneurysms described in the literature are due to congenital weakness of the fibro-muscular annuli. The majority of these patients are of African ancestry, though similar conditions have been found to a lesser degree in other race groups. Our patient had disseminated mycobacterium tuberculosis infection with cardiac involvement. There was no aneurysm noted on the initial echocardiograph, which thus excluded a congenital origin. Surgical repair of large subaortic aneurysms is the treatment of choice. They are at risk of rupture, calcification and infective endocarditis if left untreated. 


\title{
Familial component to peripartum cardiomyopathy: A case series
}

\section{Kemi Tibazarwa, Bongani Mayosi and Karen Sliwa}

Hatter Cardiovascular Research Institute, Department of Medicine, Faculty of Health Sciences, University of Cape Town, South Africa

Peri-partum cardiomyopathy (PPCM) is a form of heart failure affecting women of childbearing age, which can be associated with considerable mortality and chronic debilitating disease. Most patients present with acute post-partal heart failure that resembles the clinical presentation of idiopathic dilated cardiomyopathy. Little data is available with which to formally evaluate any genetic contribution to susceptibility to PPCM and the studies that have been published are largely case reports rather than systematic studies. There are a number of reports in the literature of PPCM in women with mothers or sisters who had the same diagnosis. Recently, however, there have been 2 reports which more strongly support the suggestion that some cases of PPCM may in fact be part of familial DCM. In one study from the Netherlands, Van SpaendonckZwarts et al. (Circulation 2010) studied 90 families with familial DCM and investigated the presence of PPCM; in addition they also examined PPCM patients and performed cardiological screening of their first-degree relatives. Their data suggest that a subset of PPCM is an initial manifestation of familial DCM and this was corroborated by the identification of a causative mutation in one family. In another study from the United States, Morales et al. (Circulation 2010) did similar observations in a large cohort study. Taken together, these findings may have important implications for cardiology screening in such families. We will report on a PPCM family screening programme performed at Groote Schuur Hospital, Cape Town and Chris Hani Baragwanath Hospital, University of the Witwatersrand, South Africa. Based on those results, general genetic testing is not recommended as a routine but is currently being done as part of research projects.

\section{Myomegalin is a novel A-kinase anchoring protein involved in the phosphorylation of cardiac myosin binding protein-C}

\author{
G.M. Uys*, A. Ramburan*, B. Loos", C.J. Kinnear*, L.J. Korkie*, J. Riedemann* and J.C. Moolman-Smook* \\ "University of Stellenbosch Medical Research Council (US/MRC), Centre for Molecular and Cellular Biology, Department of Biomedical \\ Sciences, University of Stellenbosch, South Africa \\ \#Central Analytical Facility, Department of Physiology, University of Stellenbosch, South Africa
}

Cardiac contractility is regulated by dynamic phosphorylation of sarcomeric proteins by kinases anchored close to their targets, including myosin binding protein-C (cMyBPC) and cardiac troponin I (cTNI), two proteins implicated in the inherited cardiac disease, hypertrophic cardiomyopathy.

During a yeast 2-hybrid library screen, we found that isoform 4 of myomegalin (MMGL), a known phosphodiesterase 4D-interactor, binds to the $\mathrm{N}$-terminal CMyBPC region. We show that MMGL isoform 4 interacts with PKA regulatory subunits RIA and R2A, as well as with other PKA-targets, viz. CARP, COMMD4, ENOI, ENO3 and CTNI, and that interaction with the latter increases during B-adrenergic stress. Moreover, siRNA-mediated knockdown of MMGL leads to reduction of CMyBPC levels under conditions of adrenergic stress, indicating that MMGLassisted phosphorylation is requisite for protection of $\mathrm{CMyBPC}$ against proteolytic cleavage.

Thus, this study demonstrates that MMGL isoform 4 meets all criteria for classification as an AKAP. Moreover, as it is involved in the phosphorylation of CMyBPC, as well as additional sarcomeric proteins including CTNI, MMGL is an important regulator of cardiac contractility, This has further implications for understanding the patho-aetiology of CMyBPC-and CTNI-induced HCM, and raises the question of whether MMGL might itself be considered a candidate HCM-causing or -modifying factor. 
First case of a transcatheter aortic valve implantation (TAVI) in a patient with an anomalous origin of the right coronary artery

\author{
Hellmuth Weich*, Christelle Ackermann", Hofmeyer Viljoen" and Anton Doubell* \\ "Division of Cardiology, Department of Medicine, University of Stellenbosch and Tygerberg Hospital, South Africa \\ \#Department of Radiodiagnosis, University of Stellenbosch and Tygerberg Hospital, South Africa
}

We describe the first case of placement of a transcatheter aortic valve in a man, 77 years old with an anomalous origin of his right coronary artery from the left coronary cusp. From here it follows an anterior (inter-arterial) course to the right. This would obviously be at risk for compression if a device was inflated inside the aorta.

$\mathrm{He}$ also had severe aortic stenosis with a mean gradient over the valve of $62 \mathrm{mmHg}$ and a valve area of $0.7 \mathrm{~cm}^{2}$. He had NYHA 3 symptoms as well as regular angina. He was turned down for conventional aortic valve replacement due to a porcelain aorta. The anomalous origin of the right coronary artery was diagnosed on routine pre-operative 40-slice multi detector CT-scan. Transoesophageal echo was used to evaluate the size of his aortic annulus and the bulk of calcification on the left coronary cusp was assessed. It was felt that this was unlikely to cause excessive compression of the surrounding structures. A $26 \mathrm{~mm}$ Edwards SAPIEN transcatheter valve was placed via a transapical approach without complication and a supra aortic contrast injection confirmed flow down the right coronary. Immediate post procedure ECG revealed inferior ST depression but the patient denied chest pain. 12 hours later the ECG was back to normal and the cardiac troponin levels did not rise. Two months later he was asymptomatic and repeat CT-scan confirmed a patent right coronary artery.

\title{
Initial experience with the Edwards SAPIEN transcatheter aortic valve device: The Western Cape experience
}

\author{
Hellmuth Weich*, Thomas Mabin", Wynand van Zyl\#, Jacques van Wyk ${ }^{\dagger}$ and Rocco Vivierst \\ *Division of Cardiology, Department of Medicine, University of Stellenbosch and Tygerberg Hospital, South Africa \\ \#Vergelegen Medi Clinic, Somerset West, South Africa \\ tPanorama Medi Clinic, Cape Town, South Africa
}

We wish to report our initial experience with the Edwards SAPIEN transcatheter aortic valve prosthesis in a group of 19 patients with severe aortic stenosis considered too high risk for conventional aortic valve replacement. The team consisting of 2 cardiologists, two cardiothoracic surgeons, an anaesthetist and an echo specialist evaluated the patients and performed the procedures. To qualify, all patients had to have symptomatic severe aortic stenosis with an aortic valve area of $<0.7 \mathrm{~cm}^{2}$. They had to be considered high risk for conventional surgery (logistic euro SCORE of $>20$ and/ or STS score >10) or have a contra-indication for conventional surgery (e.g. porcelain aorta).

19 procedures were performed (I I via transapical route and 9 via transfemoral route). The average logistic EuroSCORE and NYHA class was 28 and 3.47 respectively. The patients' average valve area was $0.55 \mathrm{~cm}^{2}$. The device was placed successfully in all patients although in one patient transfemoral route had to be changed to transapical intra-operatively due to inaccessible ileac vessels. The prosthesis functioned well in 100\% of cases with no coronary obstruction and aortic regurgitation of mild grade at most. At one month post procedure all patients were alive, the average NYHA class was 2.1 and the gradient across the valve came down from 52 to $12 \mathrm{mmHg}$.

Specific complications are discussed. Although small in numbers, this study shows the importance of the "heart valve team" working together. 